November 12, 2001

\title{
The wreath product principle for ordered semigroups
}

\author{
Jean-Eric Pin* and Pascal Weil ${ }^{\dagger}$ \\ Jean-Eric.Pin@liafa.jussieu.fr, Pascal.Weil@labri.fr ${ }^{\ddagger}$
}

\begin{abstract}
Straubing's wreath product principle provides a description of the languages recognized by the wreath product of two monoids. A similar principle for ordered semigroups is given in this paper. Applications to language theory extend standard results of the theory of varieties to positive varieties. They include a characterization of positive locally testable languages and syntactic descriptions of the operations $L \rightarrow L a$ and $L \rightarrow L a A^{*}$. Next we turn to concatenation hierarchies. It was shown by Straubing that the $n$-th level $\mathbf{B}_{n}$ of the dot-depth hierarchy is the variety $\mathbf{V}_{n} * \mathbf{L I}$, where $\mathbf{L I}$ is the variety of locally trivial semigroups and $\mathbf{V}_{n}$ is the $n$-th level of the Straubing-Thérien hierarchy. We prove that a similar result holds for the half levels. It follows in particular that a level or a half level of the dot-depth hierarchy is decidable if and only if the corresponding level of the Straubing-Thérien hierarchy is decidable.
\end{abstract}

\section{Introduction}

All semigroups and monoids considered in this paper are either finite or free. The reader is refered to $[13,17,15]$ for basic definitions and notations on ordered semigroups.

Straubing's "wreath product principle" $[19,21]$ provides a description of the languages recognized by the wreath product of two monoids. It has numerous applications, including Schützenberger's characterization of star-free

\footnotetext{
*LIAFA, Université Paris VII and CNRS, Case 7014, 2 Place Jussieu, 75251 Paris Cedex 05, France

${ }^{\dagger}$ LaBRI, Université Bordeaux I and CNRS, 351 cours de la Libération, 33405 Talence Cedex, France

${ }^{\ddagger}$ Work supported by INTAS project 1224 .
} 
languages $[3,11]$, the characterization of languages recognized by solvable groups [19] or the expressive power of fragments of temporal logic [4, 22].

Our aim is to develop a similar principle for ordered semigroups. The semidirect product and the wreath product of ordered semigroups are defined and studied in the authors' paper [17]. This study is supplemented in [15] by more technical results involving ordered categories. In the present paper, we establish an ordered version of the wreath product principle and derive some applications to language theory. Our main goal is to extend standard results of the theory of varieties $[5,14]$ to positive varieties. In particular, we give syntactic descriptions of the operations $L \rightarrow L a$ and $L \rightarrow L a A^{*}$ and we give a syntactic characterization of the positive locally testable languages, the "positive" counterpart of a standard result found independently by Mc Naughton [10] and Brzozowski and Simon [2].

The paper culminates with the study of the concatenation hierarchies. Recall that concatenation product and union form the so-called polynomial operations on languages. Roughly speaking, concatenation hierarchies of recognizable languages are defined by alternating the use of the polynomial closure and of the boolean closure. Two important hierarchies are the Straubing-Thérien hierarchy, whose starting point is the trivial *-variety and the dot-depth hierarchy, whose starting point is the trivial +-variety. One of the most important open problems in the theory of recognizable languages is to determine whether these hierarchies are decidable.

We first give some new results on languages of dot-depth $1 / 2$. Next, we revisit a nice result of Straubing which relates, level by level, the dot-depth hierarchy and the Straubing-Thérien hierarchy. More precisely, it was shown by Straubing that the $n$-th level $\mathbf{B}_{n}$ of the dot-depth hierarchy is the variety $\mathbf{V}_{n} * \mathbf{L I}$, where $\mathbf{L I}$ is the variety of locally trivial semigroups and $\mathbf{V}_{n}$ is the $n$-th level of the Straubing-Thérien hierarchy. We prove that a similar result holds for the half levels. It follows in particular that a level or a half level of the dot-depth hierarchy is decidable if and only if the corresponding level of the Straubing-Thérien hierarchy is decidable.

Technically speaking, we follow Straubing's main arguments, but there are a few subtleties. For instance, it is necessary to work with ordered alphabets. This is reminiscent of the study of semidirect products of profinite semigroups [1], in which profinite alphabets were required. There are also a number of new definitions (ordered automata, ordered sequential transducers, etc.) that would deserve further developments. Finally, in the section devoted to the dot-depth hierarchy, some of the arguments of [20] have been slightly simplified.

The paper is organized as follows. Section 2 introduces some new defi- 
nitions and surveys the various notions of recognition. The wreath product principle is the main topic of section 3. Operations on languages are studied in section 4 and the final section 5 is devoted to concatenation hierarchies.

\section{Recognizing subsets of an ordered semigroup}

In this section we give various definitions of recognizing processes. We define successively recognition by a morphism, by an ordered semigroup, by an ordered transformation semigroup and by an ordered deterministic automaton. We refer the reader to [17] for basic definitions on ordered semigroups and ordered transformation semigroups, their divisions and their varieties.

\subsection{Free ordered semigroups}

Let $A$ be an alphabet. We denote by $A^{+}\left(\right.$resp. $A^{*}$ ) the free semigroup (resp. free monoid) on $A$. Any partial order $\leq$ on $A$ defines a stable order on $A^{+}$ (resp. $A^{*}$ ) as follows: $u \leq v$ if and only if $u=a_{1} a_{2} \cdots a_{n}, v=b_{1} b_{2} \cdots b_{n}$, and $a_{i} \leq b_{i}$ for $1 \leq i \leq n$. In particular, words of different length are incomparable in this order. We shall denote by $\left(A^{+}, \leq\right)\left(\operatorname{resp} .\left(A^{*}, \leq\right)\right)$ the corresponding ordered semigroup (monoid). It is the free ordered semigroup over $(A, \leq)$, since it satisfies the following universal property:

Proposition 2.1 Let $(S, \leq)$ be an ordered semigroup. If $\varphi$ is an order preserving function from $(A, \leq)$ into $(S, \leq)$, there exists a unique morphism of ordered semigroups $\bar{\varphi}$ from $\left(A^{+}, \leq\right)$into $(S, \leq)$ such that $\varphi(a)=\bar{\varphi}(a)$ for every $a \in A$. Moreover $\bar{\varphi}$ is surjective if and only if $\varphi(A)$ is a set of generators for $S$.

The free ordered monoid $\left(A^{*}, \leq\right)$ over $(A, \leq)$ is defined analogously. The subsets of a free ordered semigroup (resp. monoid) are called ordered languages.

Remark. Other types of ordered semigroup structures can be considered on the free semigroup $A^{+}$. For instance, the subword order $\left(u \leq_{s} v\right.$ if $v$ is a subword $^{1}$ of $\left.u\right)$ is a stable order, but $\left(A^{+}, \leq_{s}\right)$ is not a free ordered semigroup in the sense defined here.

It is convenient to have a proper name for boolean combinations of sets involving no complement. By analogy with formal logic, where a positive formula is a formula without negation, we call these boolean combinations

\footnotetext{
${ }^{1}$ The word "subword" is used to mean a subsequence, not a segment.
} 
positive. For the formal definition, consider a set $E$. A set of subsets of $E$, which is closed under finite intersection and finite union, is called a positive boolean algebra. Note that a positive boolean algebra always contains the empty set and the full set $E$ since $\emptyset=\bigcup_{i \in \emptyset} E_{i}$ and $E=\bigcap_{i \in \emptyset} E_{i}$. The intersection of all positive boolean algebras containing a set $\mathcal{F}$ of subsets of $E$ is again a positive boolean algebra, called the positive boolean algebra generated by $\mathcal{F}$ and its elements are said to be positive boolean combinations of subsets of $\mathcal{F}$. Finally, a positive boolean algebra closed under complement is a boolean algebra.

\subsection{Recognition by ordered semigroups and ordered trans- formation semigroups.}

Let $\varphi: R \rightarrow S$ be a morphism of ordered semigroups. A subset $L$ of $R$ is recognized by $\varphi$ if there exists an order ideal $I$ of $S$ such that

$$
L=\varphi^{-1}(I)
$$

In this case, $L$ is an order ideal of $R$ and $\varphi(L)=\varphi \varphi^{-1}(I)=I$. By extension, a subset $L$ of $R$ is said to be recognized by an ordered semigroup $S$ if there exists a morphism of ordered semigroups from $R$ onto $S$ that recognizes $L$.

Let $(P, S)$ be an ordered transformation semigroup. A subset $L$ of $R$ is recognized by $(P, S)$ if there exists a morphism of ordered semigroups $\varphi: R \rightarrow S$, a state $p_{0} \in P$, the initial state, an order ideal $F \subseteq P$, the set of final states, such that

$$
L=\left\{r \in R \mid p_{0} \cdot \varphi(r) \in F\right\}
$$

Again, this implies that $L$ is an order ideal of $R$.

This definition applies in particular to ordered languages. The next two lemmas, which will be used in section 4 , will serve as examples. Recall that $U_{1}^{+}$(resp. $U_{1}^{-}$) denotes the ordered monoid $\{0,1\}$, equipped with the natural order $0 \leq 1$ (resp. $1 \leq 0$ ) and the usual product on integers. The monoid $U_{1}$ denotes the same monoid, but with the equality as an order.

Lemma 2.2 Let $(A, \leq)$ be an ordered alphabet and let $C$ be an order ideal of $A$. Then $A^{*} C A^{*}$ is an order ideal of $\left(A^{*}, \leq\right)$ recognized by $U_{1}^{+}$.

Proof. Let $\varphi:\left(A^{*}, \leq\right) \rightarrow U_{1}^{+}$be the morphism of ordered monoids defined for each letter $a \in A$ by

$$
\varphi(a)= \begin{cases}0 & \text { if } a \in C \\ 1 & \text { otherwise }\end{cases}
$$


Then $L=\varphi^{-1}(0)$.

Let $B(1,2)=\{x, y\}$ be the semigroup defined by $x x=y x=x$ and $x y=y y=y$. Letting $x \leq y$ defines a stable order on $B(1,2)$, and we let $R_{2}=(B(1,2), \leq)$ be the resulting ordered semigroup.

Lemma 2.3 Let $(A, \leq)$ be an ordered alphabet and let $C$ be an order ideal of $A$. Then $A^{*} C$ is an order ideal of $\left(A^{+}, \leq\right)$recognized by $R_{2}$.

Proof. Let $\varphi:\left(A^{+}, \leq\right) \rightarrow R_{2}$ be the morphism of ordered semigroups defined for each letter $a \in A$ by

$$
\varphi(a)= \begin{cases}x & \text { if } a \in C \\ y & \text { otherwise }\end{cases}
$$

Then $A^{*} C=\varphi^{-1}(x)$.

The case where $R$ is a free semigroup, ordered with the equality relation, is of special interest to us. The next proposition relates division and recognizability and can be proved as in the unordered case.

Proposition 2.4 If a language $L$ of $A^{+}$is recognized by $(P, S)$ and if $(P, S)$ divides $(Q, T)$, then $L$ is recognized by $(Q, T)$.

It is important to note that Proposition 2.4 cannot be extended to free ordered semigroups. For instance, Lemma 2.2 shows that, if $A=\{a, b\}$ is ordered by $a<b$, then the order ideal $A^{*} a A^{*}$ of $\left(A^{*}, \leq\right)$ is recognized by $U_{1}^{+}$. Now, $U_{1}^{+}$is a quotient of $U_{1}$, but $A^{*} a A^{*}$ is not recognized by $U_{1}$. Indeed, if $\varphi:\left(A^{+}, \leq\right) \rightarrow U_{1}$ is a morphism of ordered semigroups, then $a \leq b$ implies $\varphi(a)=\varphi(b)$, since the order on $U_{1}$ is the equality relation.

The next proposition establishes the connection between the two definitions of recognition above.

Proposition 2.5 Let $S$ be an ordered semigroup and let $(Q, T)$ be an ordered transformation semigroup.

(1) Every subset of $S$ recognized by $(Q, T)$ is recognized by $T$.

(2) A subset of $S$ is recognized by $\left(T^{1}, T\right)$ if and only if it is recognized by $T$.

(3) Every subset of $S$ recognized by $T$ is a positive boolean combination of subsets of $S$ recognized by $(Q, T)$. 
Proof. The proofs of (1) and (2), which are similar to the non-ordered case, are left to the reader. We just prove (3).

Assume that $L$ is recognized by $T$. We claim that

$$
L=\bigcup_{t \in I} \bigcap_{q \in Q}\{s \in S \mid q \cdot \varphi(s) \leq q \cdot t\}
$$

Let $R$ be the right hand side of (1). If $s \in L$ and $t=\varphi(s)$, then $t \in I$ and, for every $q \in Q, q \cdot \varphi(s) \leq q \cdot t$. Thus $L \subseteq R$. If $s \in R$, there exists $t \in I$ such that, for all $q \in Q, q \cdot \varphi(s) \leq q \cdot t$. Since $T$ acts faithfully on $Q$, it follows $\varphi(s) \leq t$ and thus $\varphi(s) \in I$ and $s \in L$, proving (1).

Now every set of the form $\{s \in S \mid q \cdot \varphi(s) \leq q \cdot t\}$ is recognized by $(Q, T)$, with $q$ as initial state and $\downarrow(q \cdot t)$ as set of final states. Thus $L$ is a positive boolean combination of subsets of $S$ recognized by $(Q, T)$.

\section{$2.3 \quad$ Ordered automata}

When subsets of a free ordered semigroup or monoid are under consideration, another mode of recognition can be used. We will treat the case of a free ordered semigroup, but the definitions could be readily adapted to the case of a free ordered monoid.

An ordered deterministic automaton is a finite deterministic automaton

$$
\mathcal{A}=\left(Q, A, \cdot, q_{0}, F\right)
$$

equipped with a partial order on $Q$ and a partial order on $A$ (both denoted $\leq$ ) satisfying the following conditions, for every $p, q \in Q$ and every $a, b \in A$ :

(1) if $p \leq q$ and if $q \cdot a$ is defined, then $p \cdot a$ is defined and $p \cdot a \leq q \cdot a$,

(2) if $a \leq b$ and if $p \cdot b$ is defined, then $p \cdot a$ is defined and $p \cdot a \leq p \cdot b$,

(3) the set $F$ of final states is an order ideal of $Q$.

Note that Condition (2) can be immediately extended as follows. For any $u, v \in A^{+}$,

$\left(2^{\prime}\right)$ if $u \leq v$ and if $p \cdot v$ is defined, then $p \cdot u$ is defined and $p \cdot u \leq p \cdot v$, The language recognized by $\mathcal{A}$ is the set $\|\mathcal{A}\|=\left\{u \in A^{+} \mid q_{0} \cdot u \in F\right\}$.

Proposition 2.6 The language of $\left(A^{+}, \leq\right)$recognized by an ordered deterministic automaton is an order ideal. 
Proof. Let $\mathcal{A}=\left(Q, A, \cdot q_{0}, F\right)$ be an ordered deterministic automaton. Let $u \in\|\mathcal{A}\|$ and let $v \leq u$. Then $q_{0} \cdot u$ is defined and belongs to $F$. By $\left(2^{\prime}\right)$, $q_{0} \cdot v$ is defined and $q_{0} \cdot v \leq q_{0} \cdot u$. Now, by (3), $F$ is an order ideal and thus $q_{0} \cdot v \in F$. Therefore $v \in\|\mathcal{A}\|$.

Given an ordered deterministic automaton $\mathcal{A}=\left(Q, A, \cdot, q_{0}, F\right)$, define a relation $\preceq$ on $A^{+}$by setting $u \preceq v$ if and only if, for every $p \in Q$, if $p \cdot v$ is defined, then $p \cdot u$ is defined, and $p \cdot u \leq p \cdot v$.

Proposition 2.7 The relation $\preceq$ is a congruence of ordered semigroups.

Proof. First, $\preceq$ is clearly reflexive and transitive. Condition $\left(2^{\prime}\right)$ above shows that it is coarser than $\leq$. To conclude, let us show that $\preceq$ is stable. Let $u, v \in A^{+}$with $u \preceq v$, and let $a \in A$.

If $p \cdot v a$ is defined, then so is $p \cdot v$. Since $u \preceq v, p \cdot u$ is defined and $p \cdot u \leq$ $p \cdot v$. Now since $(p \cdot v) \cdot a$ is defined, Condition (1) above shows that $(p \cdot u) \cdot a$ is defined and that $(p \cdot u) \cdot a \leq(p \cdot v) \cdot a$. Therefore, $u a \preceq v a$.

If $p \cdot a v$ is defined, then $p \cdot a$ is defined and $p \cdot a v=(p \cdot a) \cdot v$. Since $u \preceq v$, $(p \cdot a) \cdot u$ is defined and $(p \cdot a) \cdot u \leq(p \cdot a) \cdot v$. Thus $a u \preceq a v$.

The quotient $A^{+} / \preceq$ is called the ordered transition semigroup of $\mathcal{A}$. Together with Proposition 2.5, the next propositions will reassure the reader that the various notions of recognition are compatible.

Proposition 2.8 The language recognized by an ordered deterministic automaton is also recognized by the ordered transition semigroup of this automaton.

Proof. Let $\mathcal{A}=\left(Q, A, \cdot, q_{0}, F\right)$ be an ordered deterministic automaton, and let $\varphi$ be the natural morphism from $\left(A^{+}, \leq\right)$onto its ordered transition semigroup $(S, \leq)$. By Proposition 2.6, $\|\mathcal{A}\|$ is an order ideal of $\left(A^{+}, \leq\right)$. It follows that $P=\varphi(\|\mathcal{A}\|)$ is an order ideal of $S$. Furthermore, we have

$$
\|\mathcal{A}\|=\left\{u \in A^{+} \mid q_{0} \cdot u \in F\right\}=\left\{u \in A^{+} \mid q_{0} \cdot \varphi(u) \in P\right\}
$$

Thus $\|\mathcal{A}\|$ is recognized by $(Q, S)$.

Proposition 2.9 A language recognized by an ordered transformation semigroup on a set $P$ is also recognized by an ordered deterministic automaton having $P$ as its set of states. 
Proof. Let $L$ be a language recognized by $(P, S)$. Then there exists a morphism of ordered semigroups $\varphi: A^{+} \rightarrow S$, a state $p_{0} \in P$, an order ideal $F \subseteq P$, such that $L=\left\{u \in A^{+} \mid p_{0} \cdot \varphi(u) \in F\right\}$. Define an ordered deterministic automaton $\mathcal{A}=\left(P, A, \cdot, p_{0}, F\right)$ by setting, for every $p \in P$ and $a \in A, p \cdot a=p \cdot \varphi(a)$. Then $\mathcal{A}$ recognizes $L$.

\subsection{Positive varieties}

We recall the definition of a positive variety given in [13].

A class of recognizable languages is a correspondence $\mathcal{C}$ which associates with each alphabet $A$ a set $\mathcal{C}\left(A^{+}\right)$of recognizable languages of $A^{+}$.

A positive variety of languages is a class of ordered recognizable languages $\mathcal{V}$ such that

(1) for every alphabet $A, \mathcal{V}\left(A^{+}\right)$is a positive boolean algebra,

(2) if $\varphi: A^{+} \rightarrow B^{+}$is a morphism of semigroups, $L \in \mathcal{V}\left(B^{+}\right)$implies $\varphi^{-1}(L) \in \mathcal{V}\left(A^{+}\right)$,

(3) if $L \in \mathcal{V}\left(A^{+}\right)$and if $a \in A$, then $a^{-1} L$ and $L a^{-1}$ are in $\mathcal{V}\left(A^{+}\right)$.

Given two positive varieties of languages $\mathcal{V}$ and $\mathcal{W}$, we write $\mathcal{V} \subseteq \mathcal{W}$ if, for each alphabet $A, \mathcal{V}\left(A^{+}\right) \subseteq \mathcal{W}\left(A^{+}\right)$.

If $\mathbf{V}$ is a variety of finite ordered semigroups, we denote by $\mathcal{V}\left(A^{+}\right)$the set of recognizable languages of $A^{+}$which are recognized by an ordered semigroup of $\mathbf{V}$. Then $\mathcal{V}$ is a positive variety of languages and the correspondence $\mathbf{V} \rightarrow \mathcal{V}$ preserves inclusion. In fact, an extension of Eilenberg's variety theorem [13] states that this defines a one-to-one onto correspondence between the varieties of finite ordered semigroups and the positive varieties of languages.

At this point, in order to avoid any confusion, let us stress that the definition of a positive variety of languages does not involve ordered alphabets. Nevertheless, the use of ordered alphabets will be mandatory in several proofs and this justifies introducing a new notation. If $(A, \leq)$ is an ordered alphabet, we denote by $\mathcal{V}\left(A^{+}, \leq\right)$the set of recognizable languages of $\left(A^{+}, \leq\right)$which are recognized by an ordered semigroup of $\mathbf{V}$. The next result follows immediately from the variety theorem.

Proposition 2.10 If $\mathcal{V}$ and $\mathcal{W}$ are two positive varieties such that $\mathcal{V} \subseteq \mathcal{W}$, then, for every ordered alphabet $A, \mathcal{V}\left(A^{+}, \leq\right) \subseteq \mathcal{W}\left(A^{+}, \leq\right)$.

We conclude this section by two propositions that illustrate the notion of a positive variety. Let $\mathbf{J}_{1}^{+}$be the variety generated by the ordered monoid 
$U_{1}^{+}$. This variety is defined by the identities $x=x^{2}, x y=y x$ and $x \leq 1$ [14].

Proposition 2.11 Let $\mathcal{V}$ be the positive variety corresponding to $\mathbf{J}_{1}^{+}$. For each ordered alphabet $(A, \leq), \mathcal{V}\left(A^{*}, \leq\right)$ is the positive boolean algebra generated by the languages of the form $A^{*}(\downarrow a) A^{*}$, where $a \in A$.

Proof. Lemma 2.2 shows that, if $C$ is an order ideal of $A, A^{*} C A^{*}$ is recognized by $U_{1}^{+}$. It follows that the positive boolean algebra generated by the languages of this form is contained in $\mathcal{V}\left(A^{*}\right)$.

Conversely, let $(M, \leq) \in \mathbf{J}_{1}^{+}$and let $\varphi:\left(A^{*}, \leq\right) \rightarrow(M, \leq)$ be a morphism of ordered monoids. Let $I$ be an order ideal of $M$ and let $L=\varphi^{-1}(I)$. Let $u \in L$ and let $c(u)$ be the set of letters occurring in $u$. For $B \subseteq A$, let

$$
L(B)=\bigcap_{a \in B} A^{*}(\downarrow a) A^{*}
$$

We claim that $L(c(u))$ is a subset of $L$. First, since $M$ is idempotent and commutative, $\varphi(u)=\prod_{b \in c(u)} \varphi(b)$. On the other hand, if $v \in L(c(u))$, then $\varphi(v)=\prod_{b \in c(v)} \varphi(b) \leq \prod_{b \in c(u)} \varphi(b)$ and thus $\varphi(v) \in I$, since $I$ is an ideal. Thus $v$ is in $L$. It follows that $L=\bigcup_{u \in L} L(c(u))$. As each $c(u)$ is a subset of $A$, this seemingly infinite union is in fact finite, and this concludes the proof.

We now return to the semigroup $B(1,2)$ and the ordered semigroup $R_{2}$ considered in Lemma 2.3. Since $R_{2}$ is a quotient of $(B(1,2),=)$ and $(B(1,2),=)$ is an ordered subsemigroup of $R_{2} \times R_{2}, R_{2}$ and $(B(1,2),=)$ generate the same variety of ordered semigroups. It is known (see [6] for instance) that this variety is the variety $\mathbf{D}_{1}$ defined by the identity $y x=x$.

Proposition 2.12 Let $\mathcal{V}$ be the positive variety corresponding to $\mathbf{D}_{1}$. For each ordered alphabet $(A, \leq), \mathcal{V}\left(A^{+}, \leq\right)$is the set of finite unions of languages of the form $A^{*}(\downarrow a)$, with $a \in A$.

Proof. Let $S$ be an ordered semigroup satisfying the identity $y x=x$, and let $\varphi:\left(A^{+}, \leq\right) \rightarrow S$ be a morphism of ordered semigroups and let $I$ be an order ideal of $S$. We claim that

$$
\varphi^{-1}(I)=\bigcup_{a \in \varphi^{-1}(I)} A^{*}(\downarrow a)
$$

Let $u \in \varphi^{-1}(I)$. Setting $u=v a$, with $a \in A$, we have $\varphi(u)=\varphi(v) \varphi(a)=$ $\varphi(a) \in I$. In particular, $a \in \varphi^{-1}(I)$ and $u \in A^{*}(\downarrow a)$. 
Conversely, let $u$ be a word in the right hand side of (2). Thus $u \in$ $A^{*}(\downarrow a)$ for some letter $a$ such that $\varphi(a) \in I$. Thus $u=v b$ with $b \leq a$ and $\varphi(u)=\varphi(v) \varphi(b)=\varphi(b) \leq \varphi(a)$. Thus $\varphi(u) \in I$ and $u \in \varphi^{-1}(I)$, proving the claim.

\section{The wreath product principle}

In this section, wreath products are used to study operations on languages. We first introduce the ordered version of a standard tool of the theory of automata, the subsequential transducers.

\subsection{Order preserving sequential functions}

A subsequential transducer is an 8-tuple $\mathcal{T}=\left(Q, A, R, q_{0}, \cdot, *, m, \rho\right)$, where $Q$ is a finite set of states, $A$ is a finite alphabet called the input alphabet, $R$ is a semigroup (possibly infinite) called the output semigroup, $q_{0} \in Q$ is the initial state, $(q, a) \mapsto q \cdot a \in Q$ and $(q, a) \mapsto q * a \in R$ are partial functions with the same domain contained in $Q \times A$, called respectively the transition function and the output function, $m \in R^{1}$ is the initial prefix and $\rho: Q \rightarrow R^{1}$ is a partial function, called the terminal function.

The transition and the output functions can be extended to partial functions $Q \times A^{*} \rightarrow Q$ (resp. $Q \times A^{*} \rightarrow R^{1}$ ) by setting, for each $u \in A^{*}$ and each $a \in A$ :

$$
\begin{aligned}
q \cdot 1 & =q & & q * 1=1 \\
q \cdot(u a) & =(q \cdot u) \cdot a & & \text { if } q \cdot u \text { and }(q \cdot u) \cdot a \text { are defined } \\
q *(u a) & =(q * u)((q \cdot u) * a) & & \text { if } q * u, q \cdot u \text { and }(q \cdot u) * a \text { are defined }
\end{aligned}
$$

To decongest this type of formulas, it is convenient to fix some priority rules on the operators. Our choice is to give highest priority to concatenation, then to dot and then to star. For instance, we write $q \cdot u a$ for $q \cdot(u a), q * u a$ for $q *(u a)$ and $q \cdot u * a$ for $(q \cdot u) * a$.

The function realized by the subsequential transducer $\mathcal{T}$ is the partial function $\varphi: A^{*} \rightarrow R^{1}$ defined by

$$
\varphi(u)=m\left(q_{0} * u\right) \rho\left(q_{0} \cdot u\right)
$$

A subsequential function is a partial function that can be realized by a subsequential transducer. 
A sequential transducer is a simplified version of subsequential transducer, one without an initial prefix or a terminal function. More precisely, it is a subsequential transducer in which the initial prefix is 1 and the terminal function maps every state to 1 . A sequential transducer $\mathcal{T}$ is written simply as a 6-tuple $\mathcal{T}=\left(Q, A, R, q_{0}, \cdot, *\right)$, and the function realized by $\mathcal{T}$ is the partial function $\varphi: A^{*} \rightarrow R^{1}$ defined by

$$
\varphi(u)=q_{0} * u
$$

A sequential function is a function that can be realized by a sequential transducer.

Let $(A, \leq)$ be an ordered alphabet and let $(R, \leq)$ be an ordered semigroup. An order-preserving subsequential transducer from $\left(A^{+}, \leq\right)$into $(R, \leq)$ is a sequential transducer $\mathcal{T}=\left(Q, A, R, q_{0}, \cdot, *, m, \rho\right)$, equipped with a partial order on $Q$, and satisfying the following conditions, for each $p, q \in Q$ and $a, b \in A$ :

(1) $\operatorname{Dom}(\rho)$ is an order ideal, and $\rho$ preserves order,

(2) If $p \leq q$ and if $q \cdot a$ is defined, then $p \cdot a$ is defined and $p \cdot a \leq q \cdot a$,

(3) If $a \leq b$ and $p \cdot b$ is defined, then $p \cdot a$ is defined and $p \cdot a \leq p \cdot b$.

(4) If $a \leq b$ and $p * b$ is defined, then $p * a$ is defined and $p * a \leq p * b$.

Proposition 3.1 The function realized by an order-preserving subsequential transducer is order-preserving.

Proof. Let $\mathcal{T}=\left(Q, A, R, q_{0}, \cdot, *, m, \rho\right)$ be an order-preserving subsequential transducer and let $\varphi: A^{*} \rightarrow R^{1}$ be the subsequential function realized by $\mathcal{T}$. Let $u, v \in A^{*}$. If $u \leq v$, then $\varphi(u)=m\left(q_{0} * u\right) \rho\left(q_{0} \cdot u\right) \leq m\left(q_{0} * v\right) \rho\left(q_{0} \cdot v\right)=$ $\varphi(v)$.

The ordered transformation semigroup of an order-preserving subsequential transducer $\mathcal{T}$ is the ordered transformation semigroup of the underlying automaton $\left(Q, A, \cdot, q_{0}, \emptyset\right)$.

The main result of this section is an ordered version of a standard result [5] on subsequential functions.

Theorem 3.2 Let $\sigma:\left(A^{+}, \leq\right) \rightarrow(R, \leq)$ be a subsequential function realized by an order-preserving subsequential transducer $\mathcal{T}$, and let $(Q, T)$ be the ordered transformation semigroup of $\mathcal{T}$. If $L$ is an order ideal of $(R, \leq)$ recognized by an ordered transformation semigroup $(P, S)$, then $\sigma^{-1}(L)$ is recognized by $(P, S) \circ(Q, T)$. 
Proof. Let $\mathcal{T}=\left(Q, A, R, q_{0}, \cdot, *, m, \rho\right)$. Since $L$ is recognized by $(P, S)$, there exist a surjective morphism $\varphi: R \rightarrow S$, a state $p_{0} \in P$ and an order ideal $F \subseteq P$ such that

$$
L=\left\{u \in R \mid p_{0} \cdot \varphi(u) \in F\right\}
$$

Let $(P, S) \circ(Q, T)=(P \times Q, W)$ and define a morphism $\psi: A^{+} \rightarrow W$ by setting

$$
(p, q) \cdot \psi(u)=(p \cdot \varphi(q * u), q \cdot u)
$$

By hypothesis, the map $q \mapsto \varphi(q * u)$ is an order-preserving function from $Q$ into $S$ and thus $\psi$ is well defined. Let

$$
I=\{(p, q) \in P \times \operatorname{Dom}(\rho) \mid p \cdot \varphi(\rho(q)) \in F\}
$$

We claim that $I$ is an order ideal of $P \times Q$. Indeed if $(p, q) \in I$ and $\left(p^{\prime}, q^{\prime}\right) \leq$ $(p, q)$, then $q^{\prime} \in \operatorname{Dom}(\rho)$ since $\operatorname{Dom}(\rho)$ is an order ideal, $\varphi\left(\rho\left(q^{\prime}\right)\right) \leq \varphi(\rho(q))$ since $\varphi$ and $\rho$ are order-preserving and finally $p^{\prime} \cdot \varphi\left(\rho\left(q^{\prime}\right)\right) \leq p \cdot \varphi(\rho(q))$ by conditions (2) and (3) of the definition of an order-preserving subsequential transducer. Furthermore, since

$$
\left(p_{0} \cdot \varphi(m), q_{0}\right) \cdot \psi(u)=\left(p_{0} \cdot \varphi(m) \varphi\left(q_{0} * u\right), q_{0} \cdot u\right)
$$

we have

$$
\begin{aligned}
\sigma^{-1}(L) & =\left\{u \in A^{+} \mid \sigma(u) \in L\right\} \\
& =\left\{u \in A^{+} \mid p_{0} \cdot \varphi(\sigma(u)) \in F\right\} \\
& =\left\{u \in A^{+} \mid p_{0} \cdot \varphi\left(m\left(q_{0} * u\right) \rho\left(q_{0} \cdot u\right)\right) \in F\right\} \\
& =\left\{u \in A^{+} \mid p_{0} \cdot \varphi(m) \varphi\left(q_{0} * u\right) \varphi\left(\rho\left(q_{0} \cdot u\right)\right) \in F\right\} \\
& =\left\{u \in A^{+} \mid\left(p_{0} \cdot \varphi(m), q_{0}\right) \cdot \psi(u) \in I\right\}
\end{aligned}
$$

Therefore, $\sigma^{-1}(L)$ is recognized by $(P \times Q, W)$.

\subsection{Languages recognized by wreath products}

In this section, $(A, \leq)$ is a fixed ordered alphabet, and $\left(A^{+}, \leq\right)$is the corresponding free ordered semigroup.

The aim of this section is to characterize the languages recognized by the wreath product of two ordered transformation semigroups. This study motivates the introduction of an auxiliary tool, the sequential transducer of a morphism. 
Let $T$ be an ordered semigroup and let $\varphi:\left(A^{+}, \leq\right) \rightarrow T$ be a surjective morphism of ordered semigroups. Set $B_{T}=T^{1} \times A$, ordered by the product order: for $(t, a),\left(t^{\prime}, a^{\prime}\right) \in B_{T}$,

$$
(t, a) \leq\left(t^{\prime}, a^{\prime}\right) \text { if and only if } t \leq t^{\prime} \text { and } a \leq a^{\prime}
$$

Let $\mathcal{T}_{\varphi}=\left(T^{1}, A, B_{T}^{+}, \cdot, *, 1\right)$ be the order-preserving sequential transducer defined, for each $s \in T^{1}$ and $a \in A$, by $s \cdot a=s \varphi(a)$ and $s * a=(s, a)$.

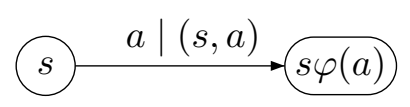

Figure 3.1: The sequential transducer $\mathcal{T}_{\varphi}$.

Note that the ordered transformation semigroup of $\mathcal{T}_{\varphi}$ is $\left(T^{1}, T\right)$. The sequential function $\sigma_{\varphi}: A^{+} \rightarrow B_{T}^{+}$defined by $\mathcal{T}_{\varphi}$ is called the sequential function associated with $\varphi$. One easily verifies that

$$
\sigma_{\varphi}\left(a_{1} a_{2} \cdots a_{n}\right)=\left(1, a_{1}\right)\left(\varphi\left(a_{1}\right), a_{2}\right) \cdots\left(\varphi\left(a_{1} \cdots a_{n-1}\right), a_{n}\right)
$$

Let $X=(P, S)$ and $Y=(Q, T)$ be two ordered transformation semigroups, let $Z=X \circ Y=(P \times Q, W)$, and let $L$ be a language of $\left(A^{+}, \leq\right)$recognized by $Z$. Then there exist a state $\left(p_{0}, q_{0}\right)$, an order ideal $F$ of $P \times Q$ and a morphism of ordered semigroups $\eta:\left(A^{+}, \leq\right) \rightarrow W$ such that $L=\left\{u \in A^{+} \mid\right.$ $\left.\left(p_{0}, q_{0}\right) \cdot \eta(u) \in F\right\}$. Denote by $\pi$ the natural projection from $W$ onto $T$, defined by $\pi(f, t)=t$ and let $\varphi=\pi \circ \eta: A^{+} \rightarrow T$.

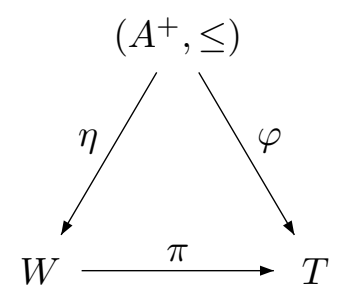

Let $B_{Q}=Q \times A$, equipped with the product order. Define a function $\sigma: A^{+} \rightarrow B_{Q}^{+}$by

$$
\sigma\left(a_{1} a_{2} \cdots a_{n}\right)=\left(q_{0}, a_{1}\right)\left(q_{0} \cdot \varphi\left(a_{1}\right), a_{2}\right) \cdots\left(\left(q_{0} \cdot \varphi\left(a_{1} \cdots a_{n-1}\right)\right), a_{n}\right)
$$

and, for each $q \in Q$, an order-preserving function $\lambda_{q}: B_{T}^{+} \rightarrow B_{Q}^{+}$by

$$
\lambda_{q}(t, a)=(q \cdot t, a)
$$

Then $\lambda_{q_{0}}$ serves as a bridge between $\sigma$ and $\sigma_{\varphi}$, since $\sigma=\lambda_{q_{0}} \circ \sigma_{\varphi}$, and we have the following diagram: 


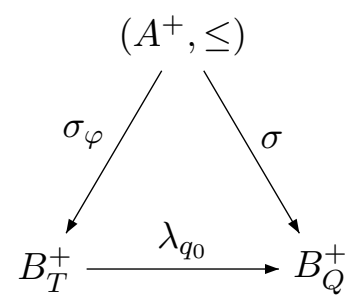

Note also that $\sigma$ is an order-preserving sequential function, realized by the transducer $\mathcal{T}_{\sigma}=\left(Q, A, B_{Q}^{+}, q_{0}, \cdot, *\right)$ where $q \cdot a=q \cdot \varphi(a)$ and $q * a=(q, a)$.

Theorem 3.3 The language $L$ is a finite union of languages of the form $U \cap \sigma_{\varphi}^{-1}(V)$, where $U \subseteq A^{+}$is recognized by $\varphi$ and $V \subseteq B_{T}^{+}$is recognized by $X$.

Proof. First, we may assume that $F$ is a principal order ideal. This is a consequence of the formula

$$
L=\bigcup_{(p, q) \in F}\left\{u \in A^{+} \mid\left(p_{0}, q_{0}\right) \cdot u \in \downarrow(p, q)\right\}
$$

Thus we may suppose that $F=\downarrow(p, q)=(\downarrow p) \times(\downarrow q)$ for some $(p, q) \in P \times Q$. For each letter $a$, set $\eta(a)=\left(f_{a}, t_{a}\right)$. Note that $\varphi(a)=t_{a}$. Define an orderpreserving function $\alpha: B_{Q} \rightarrow S$ by setting $\alpha(q, a)=q \cdot f_{a}$ and extend it into a morphism of ordered semigroups $\alpha: B_{Q}^{+} \rightarrow S$. Setting $\gamma=\lambda_{q_{0}} \circ \alpha$, we obtain the following commutative diagram:

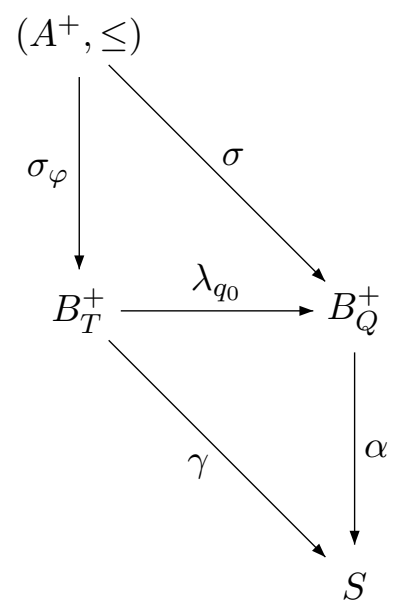


Let $u=a_{1} a_{2} \cdots a_{n}$ be a word. Then

$$
\begin{aligned}
\left(p_{0}, q_{0}\right) \cdot u & =\left(p_{0}, q_{0}\right) \cdot\left(f_{a_{1}}, t_{a_{1}}\right)\left(f_{a_{2}}, t_{a_{2}}\right) \cdots\left(f_{a_{n}}, t_{a_{n}}\right) \\
& =\left(p_{0}+q_{0} \cdot f_{a_{1}}+\cdots+\left(q_{0} \cdot t_{a_{1}} \cdots t_{a_{n-1}}\right) f_{a_{n}}, q_{0} \cdot t_{a_{1}} \cdots t_{a_{n}}\right) \\
& =\left(p_{0}+\alpha\left(q_{0}, a_{1}\right)+\cdots+\alpha\left(q_{0} \cdot \varphi\left(a_{1} \cdots a_{n-1}\right), a_{n}\right), q_{0} \cdot \varphi(u)\right) \\
& =\left(p_{0}+\alpha(\sigma(u)), q_{0} \cdot \varphi(u)\right) \\
& =\left(p_{0}+\gamma\left(\sigma_{\varphi}(u)\right), q_{0} \cdot \varphi(u)\right)
\end{aligned}
$$

It follows that $\left(p_{0}, q_{0}\right) \cdot u \in F$ if and only if the following two conditions are satisfied:

(1) $p_{0}+\gamma\left(\sigma_{\varphi}(u)\right) \leq p$

(2) $q_{0} \cdot \varphi(u) \leq q$

Setting $U=\left\{u \in A^{+} \mid q_{0} \cdot \varphi(u) \leq q\right\}$ and $V=\left\{v \in B^{+} \mid p_{0}+\gamma(v) \leq p\right\}$, condition (1) can be reformulated as $u \in \sigma_{\varphi}^{-1}(V)$, and condition (2) as $u \in U$. Thus

$$
L=U \cap \sigma_{\varphi}^{-1}(V)
$$

Now, $U$ is recognized by $\varphi$ and $V$ is recognized by $X$, which concludes the proof.

When the ordered transformation semigroups $X$ and $Y$ are both ordered semigroups, the following corollary is obtained.

Corollary 3.4 Let $(A, \leq)$ be an ordered alphabet and let $S$ and $T$ be two ordered semigroups. Every language of $\left(A^{+}, \leq\right)$recognized by $S \circ T$ is a finite union of languages of the form $U \cap \sigma_{\varphi}^{-1}(V)$, where $\varphi:\left(A^{+}, \leq\right) \rightarrow T$ is a morphism of ordered semigroups, $U \subseteq A^{+}$is recognized by $\varphi$ and $V \subseteq B_{T}^{+}$ is recognized by $S$.

We now derive a variety version of the previous theorem. It is stated in the case where both $\mathbf{V}$ and $\mathbf{W}$ are varieties of ordered monoids, but similar statements hold if $\mathbf{V}$ or $\mathbf{W}$ is a variety of ordered semigroups.

Corollary 3.5 Let $\mathbf{V}$ and $\mathbf{W}$ be two varieties of ordered monoids and let $\mathcal{U}$ be the positive variety associated with $\mathbf{V} * \mathbf{W}$. Then, for every alphabet $A, \mathcal{U}\left(A^{*}\right)$ is the smallest positive boolean algebra containing $\mathcal{W}\left(A^{*}\right)$ and the languages of the form $\sigma_{\varphi}^{-1}(V)$, where $\sigma_{\varphi}$ is the sequential function associated with a morphism $\varphi: A^{*} \rightarrow T$, with $T \in \mathbf{W}$, and $V \in \mathcal{V}\left(B_{T}^{*}, \leq\right)$. 
Proof. Since $\mathbf{W}$ is contained in $\mathbf{V} * \mathbf{W}, \mathcal{W}\left(A^{*}\right)$ is contained in $\mathcal{U}\left(A^{*}\right)$. Furthermore, if $V$ and $\sigma_{\varphi}$ are given as in the statement, then $\sigma_{\varphi}^{-1}(V) \in$ $\mathcal{U}\left(A^{*}\right)$ by Theorem 3.2.

Now, it is shown in [17] that $\mathbf{V} * \mathbf{W}$ is the class of all divisors of wreath products of the form $S \circ T$ with $S \in \mathbf{V}$ and $T \in \mathbf{W}$. It follows by Proposition 2.4 that every language of $\mathcal{U}\left(A^{*}\right)$ is recognized by such a wreath product, and Corollary 3.4 suffices to conclude.

\section{Operations on languages}

In this section, we extend standard results on varieties of languages to positive varieties. We study the operations $L \mapsto L a A^{*}$ and $L \mapsto L a$, where $a$ is a letter of $A$. Then we give a description of the languages corresponding to $\mathbf{J}_{1}^{+} * \mathbf{V}, \mathbf{J}^{+} * \mathbf{V}, \mathbf{D}_{1} * \mathbf{V}$ and $\mathbf{D} * \mathbf{V}$, where $\mathbf{V}$ is a variety of ordered monoids (resp. semigroups). We remind the reader that the definition of the variety $\mathbf{D}_{1}$ was given at the end of section 2.4.

\subsection{The operation $L \mapsto L a A^{*}$}

The study of this operation is based on the following proposition.

Proposition 4.1 Let $(A, \leq)$ be an ordered alphabet, let $a \in A$ and let $L$ be an order ideal of $\left(A^{*}, \leq\right)$ recognized by an ordered monoid $T$. Then $L(\downarrow a) A^{*}$ is recognized by the wreath product $U_{1}^{+} \circ T$.

Proof. Let $\varphi:\left(A^{*}, \leq\right) \rightarrow T$ be a morphism recognizing $L$, let $B=B_{T}$ and let $\sigma_{\varphi}: A^{*} \rightarrow B^{*}$ be the sequential function associated with $\varphi$. Let $P=\varphi(L)$ and $C=\{(p, b) \mid p \in P, b \leq a\}$. By construction, $C$ is an order ideal of $B$, and we have

$$
\begin{aligned}
\sigma_{\varphi}^{-1}\left(B^{*} C B^{*}\right) & =\left\{u \in A^{+} \mid \sigma_{\varphi}(u) \in B^{*} C B^{*}\right\} \\
& =\left\{a_{1} a_{2} \cdots a_{n} \in A^{+} \mid \exists i\left(\varphi\left(a_{1} a_{2} \cdots a_{i-1}\right), a_{i}\right) \in C\right\} \\
& =\left\{a_{1} a_{2} \cdots a_{n} \in A^{+} \mid \exists i a_{i} \leq a \text { and } a_{1} a_{2} \cdots a_{i-1} \in \varphi^{-1}(P)\right\} \\
& =L(\downarrow a) A^{*}
\end{aligned}
$$

Now, by Lemma $2.2, B^{*} C B^{*}$ is recognized by $U_{1}^{+}$, and the proposition follows from Theorem 3.2.

A similar result holds if $T$ is an ordered semigroup which is not a monoid. 
Proposition 4.2 Let $(A, \leq)$ be an ordered alphabet, let $a \in A$ and let $L$ be an order ideal of $\left(A^{+}, \leq\right)$recognized by an ordered semigroup $T$ such that $T \neq T^{1}$. Then the languages $L(\downarrow a) A^{*}$ and $(\downarrow a) A^{*}$ are recognized by the wreath product $U_{1}^{+} \circ\left(T^{1}, T\right)$.

Proof. Let $\varphi:\left(A^{+}, \leq\right) \rightarrow T$ be a morphism recognizing $L$, let $B=B_{T}$ and let $\sigma_{\varphi}: A^{+} \rightarrow B^{+}$be the sequential function associated with $\varphi$. Let

$$
P=\varphi(L), C=\{(p, b) \mid p \in P, b \leq a\} \text { and } D=\{(1, b) \mid b \leq a\} .
$$

Since $T$ is not a monoid, 1 is not comparable to any other element of $T$ in the ordered monoid $T^{1}$, and thus $C$ and $D$ are both order ideals of $B$. Since

$$
\sigma_{\varphi}\left(a_{1} a_{2} \cdots a_{n}\right)=\left(1, a_{1}\right)\left(\varphi\left(a_{1}\right), a_{2}\right) \cdots\left(\varphi\left(a_{1} a_{2} \cdots a_{n-1}\right), a_{n}\right)
$$

the word $\sigma_{\varphi}\left(a_{1} a_{2} \cdots a_{n}\right)$ contains a letter of $C$ if and only if $\varphi\left(a_{1} a_{2} \cdots a_{i-1}\right) \in$ $P$ and $a_{i} \leq a$ for some $i>1$. It contains a letter of $D$ if and only if $a_{1} \leq a$. Therefore

$$
\begin{aligned}
\sigma_{\varphi}^{-1}\left(B^{*} C B^{*}\right) & =L(\downarrow a) A^{*} \\
\sigma_{\varphi}^{-1}\left(B^{*} D B^{*}\right) & =(\downarrow a) A^{*}
\end{aligned}
$$

Now, by Lemma $2.2, B^{*} C B^{*}$ and $B^{*} D B^{*}$ are both recognized by $U_{1}^{+}$, and the proposition follows from Theorem 3.2.

The next proposition characterizes the varieties of ordered semigroups containing a semigroup which is not a monoid.

Proposition 4.3 A variety of ordered semigroups contains an ordered semigroup which is not a monoid if and only if it is not a variety of groups.

Proof. It suffices to show that if a variety $\mathbf{V}$ of ordered semigroups contains an ordered semigroup $(S, \leq)$ which is not a group, then it contains an ordered semigroup which is not a monoid. The result is clear if $S$ itself is not a monoid, so we are left with the case where $S$ is a monoid. Let $I$ be the minimal ideal of $S$. If $I$ contains two $\mathcal{R}$-equivalent (resp. $\mathcal{L}$-equivalent) idempotents $a$ and $b$, then the subsemigroup $(\{a, b\}, \leq)$ is an ordered subsemigroup of $(S, \leq)$ which is not a monoid ${ }^{2}$. Otherwise $I$ is a group, and $S \neq I$. Now since $S$ is a monoid, it admits a maximal $\mathcal{D}$-class $H$ which is a group disjoint from $I$. Let now $T$ be the subsemigroup of $S \times S$ generated by

\footnotetext{
${ }^{2}$ In fact $\{a, b\}$ is a semigroup isomorphic to $B(1,2)$ (resp. $B(2,1)$ )
} 
$((S \backslash H) \times S) \cup(S \times(S \backslash H))$. Then $T$ belongs to $\mathbf{V}$ but is not a monoid.

We now formulate Propositions 4.1 and 4.2 in terms of varieties.

Theorem 4.4 Let $\mathbf{V}$ be a variety of ordered monoids and let $\mathcal{V}$ be the corresponding positive variety. Then the positive variety $\mathcal{W}$ which corresponds to $\mathbf{J}_{\mathbf{1}}^{+} * \mathbf{V}$ is defined as follows. For each alphabet $A, \mathcal{W}\left(A^{*}\right)$ is the positive boolean algebra generated by the languages $L$ and $L a A^{*}$, where $a \in A$ and $L \in \mathcal{V}\left(A^{*}\right)$.

Proof. For each alphabet $A$, denote by $\mathcal{V}^{\prime}\left(A^{*}\right)$ the positive boolean algebra generated by the languages $L$ and $L a A^{*}$, where $a \in A$ and $L \in \mathcal{V}\left(A^{*}\right)$.

We first show that $\mathcal{V}^{\prime}\left(A^{*}\right) \subseteq \mathcal{W}\left(A^{*}\right)$. Since $\mathbf{J}_{1}^{+} * \mathbf{V}$ contains $\mathbf{V}, \mathcal{W}\left(A^{*}\right)$ contains $\mathcal{V}\left(A^{*}\right)$. Let $L \in \mathcal{V}\left(A^{*}\right)$ and $a \in A$. Then $L$ is recognized by some ordered monoid $T$ of $\mathbf{V}$ and, by Proposition $4.1, L a A^{*}$ is recognized by $U_{1}^{+} \circ T$. Now, by [17, Proposition 3.5], this ordered monoid belongs to $\mathbf{J}_{1}^{+} * \mathbf{V}$, showing that $L a A^{*} \in \mathcal{W}\left(A^{*}\right)$.

To establish the opposite inclusion, it suffices now, by Corollary 3.5, to verify that $L \in \mathcal{V}^{\prime}\left(A^{*}\right)$ for every language $L$ of the form $\sigma_{\varphi}^{-1}(V)$, where $\sigma_{\varphi}$ is the sequential function associated with a morphism of ordered monoids $\varphi: A^{*} \rightarrow T$, with $T \in \mathbf{V}$, and $V$ is an order ideal of $\left(B_{T}^{*}, \leq\right)$ recognized by an ordered monoid of $\mathbf{J}_{1}^{+}$. By Proposition 2.11, $V$ is a positive boolean combination of languages of the form $B_{T}^{*} C B_{T}^{*}$, for some order ideal $C \subseteq$ $B_{T}$. Since boolean operations commute with $\sigma_{\varphi}^{-1}$, we may assume that $V=B_{T}^{*} C B_{T}^{*}$, where $C=\downarrow(t, a)$ for some $(t, a) \in B_{T}$. In that case

$$
\begin{aligned}
L & =\left\{u \in A^{*} \mid \sigma_{\varphi}(u) \in B_{T}^{*} C B_{T}^{*}\right\} \\
& =\left\{a_{1} a_{2} \cdots a_{n} \in A^{*} \mid\left(\varphi\left(a_{1} \cdots a_{i-1}\right), a_{i}\right) \leq(t, a) \text { for some } i\right\} \\
& =\varphi^{-1}(\downarrow t) a A^{*}
\end{aligned}
$$

Now, $\varphi^{-1}(\downarrow t)$ is recognized by $T$ and thus $\varphi^{-1}(\downarrow t) \in \mathcal{V}\left(A^{*}\right)$. It follows that $\varphi^{-1}(\downarrow t) a A^{*} \in \mathcal{V}^{\prime}\left(A^{*}\right)$ and thus $L \in \mathcal{V}^{\prime}\left(A^{*}\right)$. Therefore $\mathcal{W}\left(A^{*}\right) \subseteq$ $\mathcal{V}^{\prime}\left(A^{*}\right)$.

The semigroup version of Theorem 4.4 can be obtained by using Proposition 4.2 instead of Proposition 4.1.

Theorem 4.5 Let $\mathbf{V}$ be a variety of ordered semigroups which is not a variety of groups, and let $\mathcal{V}$ be the corresponding positive variety. Then the positive variety $\mathcal{W}$ which corresponds to $\mathbf{J}_{\mathbf{1}}^{+} * \mathbf{V}$ is defined as follows. 
For each alphabet $A, \mathcal{W}\left(A^{+}\right)$is the positive boolean algebra generated by the languages $L, a A^{*}$ and $L a A^{*}$, where $a \in A$ and $L \in \mathcal{V}\left(A^{+}\right)$.

Using the same techniques, one could prove the following result, whose proof is left to the reader. In this statement, $L^{c}$ denotes the complement (in $A^{*}$ ) of a language $L$ of $A^{*}$.

Theorem 4.6 Let $\mathbf{V}$ be a variety of ordered monoids and let $\mathcal{V}$ be the corresponding positive variety. Then the positive variety $\mathcal{W}$ which corresponds to $\mathbf{J}_{\mathbf{1}} * \mathbf{V}$ is defined as follows. For each alphabet $A, \mathcal{W}\left(A^{*}\right)$ is the positive boolean algebra generated by the languages $L, L a A^{*}$ and $\left(L^{c} a A^{*}\right)^{c}$, where $a \in A$ and $L \in \mathcal{V}\left(A^{*}\right)$.

Of course, if $\mathbf{V}$ is a variety of monoids, we recover the well known result [5] that the syntactic monoid of a language belongs to $\mathbf{J}_{\mathbf{1}} * \mathbf{V}$ if and only if it belongs to the boolean algebra generated by the languages $L$ or $L a A^{*}$, where $a \in A$ and $L \in \mathcal{V}\left(A^{*}\right)$.

Another consequence of Theorem 4.4 is worth stating.

Proposition 4.7 Let $\mathbf{V}$ be a variety of ordered monoids and let $\mathcal{V}$ be the corresponding positive variety. Then the positive variety $\mathcal{W}$ corresponding to $\mathbf{J}^{+} * \mathbf{V}$ is defined as follows. For each alphabet $A, \mathcal{W}\left(A^{*}\right)$ is the smallest positive boolean algebra containing $\mathcal{V}\left(A^{*}\right)$ and closed under the operation $L \mapsto L a A^{*}$, for each $a \in A$.

Proof. Indeed, it is shown in [17] that $\mathbf{J}^{+}$is the smallest variety of ordered monoids closed under wreath product and containing $U_{1}^{+}$.

A semigroup version also holds.

Proposition 4.8 Let $\mathbf{V}$ be a variety of ordered semigroups which is not a variety of groups and let $\mathcal{V}$ be the corresponding positive variety. Then the positive variety $\mathcal{W}$ corresponding to $\mathbf{J}^{+} * \mathbf{V}$ is defined as follows. For each alphabet $A, \mathcal{W}\left(A^{+}\right)$is the smallest positive boolean algebra containing $\mathcal{V}\left(A^{+}\right)$and the languages $a A^{*}$, for $a \in A$, and closed under the operation $L \mapsto L a A^{*}$, for each $a \in A$.

\subsection{The operation $L \mapsto L a$}

The results are quite similar to those presented in subsection 4.1 , but the ordered monoid $U_{1}^{+}$is now replaced by the ordered semigroup $R_{2}$. The next result is adapted from [20, Lemma 9.8]. 
Proposition 4.9 Let $(A, \leq)$ be an ordered alphabet, let $a \in A$ and let $L$ be an order ideal of $\left(A^{*}, \leq\right)$ recognized by an ordered monoid $T$. Then $L(\downarrow a)$ is recognized by the wreath product $R_{2} \circ T$.

Proof. Let $\varphi:\left(A^{*}, \leq\right) \rightarrow T$ be a morphism recognizing $L$, let $B=B_{T}$ and let $\sigma_{\varphi}: A^{*} \rightarrow B^{*}$ be the sequential function associated with $\varphi$. Let $P=\varphi(L)$ and $C=\{(p, b) \mid p \in P, b \leq a\}$. By construction, $C$ is an order ideal of $B$, and we have

$$
\begin{aligned}
\sigma_{\varphi}^{-1}\left(B^{*} C\right) & =\left\{u \in A^{*} \mid \sigma_{\varphi}(u) \in B^{*} C\right\} \\
& =\left\{a_{1} a_{2} \cdots a_{n} \in A^{+} \mid\left(\varphi\left(a_{1} a_{2} \cdots a_{n-1}\right), a_{n}\right) \in C\right\} \\
& =\left\{a_{1} a_{2} \cdots a_{n} \in A^{+} \mid a_{n} \leq a \text { and } a_{1} a_{2} \cdots a_{n-1} \in \varphi^{-1}(P)\right\} \\
& =L(\downarrow a)
\end{aligned}
$$

By Lemma 2.3, the language $B^{*} C$ is recognized by $R_{2}$. The proposition now follows from Theorem 3.2.

A result similar to Proposition 4.2 holds if $T$ is an ordered semigroup which is not a monoid. It suffices to modify the proof of Proposition 4.9 in the same way as we modified the proof of Proposition 4.1 to obtain Proposition 4.2 .

Proposition 4.10 Let $(A, \leq)$ be an ordered alphabet, let $a \in A$ and let $L$ be an order ideal of $\left(A^{+}, \leq\right)$recognized by an ordered semigroup $T$ such that $T \neq T^{1}$. Then the languages $L(\downarrow a)$ and $\downarrow$ a are recognized by the wreath product $R_{2} \circ\left(T^{1}, T\right)$.

We now turn to varieties. Recall that the ordered semigroup $R_{2}$ generates the variety $\mathbf{D}_{1}$.

Theorem 4.11 Let $\mathbf{V}$ be a variety of ordered monoids and let $\mathcal{V}$ be the corresponding positive variety. Let $\mathcal{W}$ be the positive variety corresponding to $\mathbf{D}_{1} * \mathbf{V}$. Then, for each alphabet $A, \mathcal{W}\left(A^{+}\right)$is the positive boolean algebra generated by the languages $L$ (contained in $A^{+}$) or La, where $a \in A$ and $L \in \mathcal{V}\left(A^{*}\right)$.

Proof. For each alphabet $A$, let $\mathcal{V}^{\prime}\left(A^{+}\right)$be the positive boolean algebra generated by the languages $L$ (contained in $A^{+}$) or $L a$, where $a \in A$ and $L \in \mathcal{V}\left(A^{*}\right)$.

We first show that $\mathcal{V}^{\prime}\left(A^{+}\right) \subseteq \mathcal{W}\left(A^{+}\right)$. Since $\mathbf{D}_{1} * \mathbf{V}$ contains $\mathbf{V}$, every language of $\mathcal{V}\left(A^{*}\right)$ contained in $A^{+}$is also in $\mathcal{W}\left(A^{+}\right)$. Let $L \in \mathcal{V}\left(A^{*}\right)$ and 
$a \in A$. Then $L$ is recognized by some ordered monoid $T$ of $\mathbf{V}$ and, by Proposition 4.9, $L a$ is recognized by $R_{2} \circ T$. Now, by [17, Proposition 3.5], this semigroup belongs to $\mathbf{D}_{1} * \mathbf{V}$. Therefore $L a \in \mathcal{W}\left(A^{+}\right)$.

To establish the opposite inclusion, it suffices now, by Corollary 3.5, to verify that $L \in \mathcal{V}^{\prime}\left(A^{+}\right)$for every language $L$ of the form $\sigma_{\varphi}^{-1}(V)$, where $\sigma_{\varphi}$ is the sequential function associated with a morphism of ordered monoids $\varphi: A^{*} \rightarrow T$, with $T \in \mathbf{V}$, and $V$ is an order ideal of $\left(B_{T}^{+}, \leq\right)$recognized by an ordered semigroup of $\llbracket y x=x \rrbracket$. By Proposition $2.12, V$ is a finite union of languages of the form $B_{T}^{*}(\downarrow c)$, for some letter $c \in B_{T}$. Since union commutes with $\sigma_{\varphi}^{-1}$, we may assume that $V=B_{T}^{*}(\downarrow c)$, where $c=(t, a)$ for some $(t, a) \in B_{T}$. In that case

$$
\begin{aligned}
L & =\left\{u \in A^{+} \mid \sigma_{\varphi}(u) \in B_{T}^{*} c\right\} \\
& =\left\{a_{1} a_{2} \cdots a_{n} \in A^{+} \mid\left(\varphi\left(a_{1} \cdots a_{n-1}\right), a_{n}\right) \leq(t, a)\right\} \\
& =\varphi^{-1}(\downarrow t) a
\end{aligned}
$$

Now, $\varphi^{-1}(\downarrow t)$ is recognized by $T$ and thus $\varphi^{-1}(\downarrow t) \in \mathcal{V}\left(A^{*}\right)$. It follows that $\varphi^{-1}(\downarrow t) a \in \mathcal{V}^{\prime}\left(A^{+}\right)$for each letter $a$ and thus $L \in \mathcal{V}^{\prime}\left(A^{+}\right)$. Therefore $\mathcal{W}\left(A^{+}\right) \subseteq \mathcal{V}^{\prime}\left(A^{+}\right)$.

The semigroup version of Theorem 4.11 can be obtained by using Proposition 4.10 instead of Proposition 4.9.

Theorem 4.12 Let $\mathbf{V}$ be a variety of ordered semigroups which is not a variety of groups, and let $\mathcal{V}$ be the corresponding positive variety. Let $\mathcal{W}$ be the positive variety corresponding to $\llbracket y x=x \rrbracket * \mathbf{V}$. Then, for each alphabet $A, \mathcal{W}\left(A^{+}\right)$is the positive boolean algebra generated by the languages $L,\{a\}$ or La, where $a \in A$ and $L \in \mathcal{V}\left(A^{+}\right)$.

The smallest variety of semigroups containing $B(1,2)$ and closed under semidirect product is known to be the variety $\mathbf{D}[5]$. Therefore, Theorems 4.11 and 4.12 lead to a language interpretation of the operation $\mathbf{V} \rightarrow \mathbf{D} * \mathbf{V}$.

Corollary 4.13 Let $\mathrm{V}$ be a variety of ordered monoids and let $\mathcal{V}$ be the corresponding positive variety. Let $\mathcal{W}$ be the positive variety corresponding to $\mathbf{D} * \mathbf{V}$. Then, for each alphabet $A, \mathcal{W}\left(A^{+}\right)$is the smallest positive boolean algebra containing $\mathcal{V}\left(A^{*}\right)$ and closed under the operation $L \mapsto L u$, for each $u \in A^{*}$.

Corollary 4.14 Let V be a variety of ordered semigroups which is not a variety of groups and let $\mathcal{V}$ be the corresponding positive variety. Let $\mathcal{W}$ 
be the positive variety corresponding to $\mathbf{D} * \mathbf{V}$. Then, for each alphabet $A$, $\mathcal{W}\left(A^{+}\right)$is the smallest positive boolean algebra containing $\mathcal{V}\left(A^{+}\right)$and the languages $\{a\}$, for each $a \in A$, and closed under the operation $L \mapsto L u$, for each $u \in A^{*}$.

\subsection{Locally testable languages}

Let $A$ be an alphabet and let $u$ be a word of length $\geq n$ of $A^{+}$. We denote by $p_{n}(u)$ (resp. $s_{n}(u)$ ) the prefix (resp. suffix) of length $n$ of $u$. We also denote by $F_{n}(u)$ the set of all factors of length $n$ of $u$.

A language is prefix $k$-testable if it is saturated for the equivalence relation $\sim_{k}$ defined by $u \sim_{k} v$ if and only if $u=v$ or $|u|,|v| \geq k$ and $p_{k}(u)=p_{k}(v)$. Equivalently, a language is prefix $k$-testable if and only if it is a finite union of languages of the form $\{u\}$ or $p A^{*}$, with $|u|<k$ and $|p|=k$. The definition of a suffix $k$-testable language is dual.

The syntactic characterization of these classes is well-known [12, Exercise 3.2]. Let $\mathbf{K}_{k}$ (resp. $\mathbf{D}_{k}$ ) denote the variety of semigroups defined by the identity $x_{1} \cdots x_{k} y=x_{1} \cdots x_{k}$ (resp. $y x_{1} \cdots x_{k}=x_{1} \cdots x_{k}$ ).

\section{Proposition 4.15}

(1) A language is prefix $k$-testable if and only if its syntactic semigroup belongs to $\mathbf{K}_{k}$.

(2) A language is suffix $k$-testable if and only if its syntactic semigroup belongs to $\mathbf{D}_{k}$.

A language is prefix-suffix $k$-testable if it is saturated for the equivalence relation $\sim_{k}$ defined by $u \sim_{k} v$ if and only if $u=v$ or $|u|,|v| \geq k$ and

(1) $p_{k}(u)=p_{k}(v)$,

(2) $s_{k}(u)=s_{k}(v)$.

The $\sim_{k}$-class of a word $u$ is equal to $\{u\}$ if $|u|<k$ and to $p_{k}(u) A^{*} \cap A^{*} s_{k}(u)$ otherwise.

Proposition 4.16 Let $L$ be a language of $A^{+}$. The following conditions are equivalent:

(1) $L$ is prefix-suffix $k$-testable,

(2) $L$ is a positive boolean combination of languages of the form $\{u\}, p A^{*}$ or $A^{*} s$, with $|u|<k$ and $|p|=|s|=k$,

(3) $L$ is a finite union of languages of the form $\{u\}$ or $p A^{*} \cap A^{*} s$, with $|u|<k$ and $|p|=|s|=k$. 
Proof. The equivalence of (1) and (3) follows from the description of the $\sim_{k}$-class of a word $u$.

(3) implies (2) is trivial. To show that (2) implies (1), it suffices to observe that each of the languages $\{u\}, p A^{*}$ or $A^{*} s$, with $|u|<k$ and $|p|=|s|=k$, is saturated for $\sim_{k}$.

A language is positively $k$-testable if it is an order ideal for the relation $\leq_{k}$ defined by $v \leq_{k} u$ if and only if $u=v$ or $|u|,|v| \geq k$ and

(1) $p_{k-1}(u)=p_{k-1}(v)$,

(2) $s_{k-1}(u)=s_{k-1}(v)$,

(3) $F_{k}(u) \subseteq F_{k}(v)$.

Proposition 4.17 A language is positively $k$-testable if and only if it is a positive boolean combination of languages of the form $\{u\}, v A^{*}, A^{*} v$ or $A^{*} w A^{*}$, with $|u|<k-1,|v|=k-1$ and $|w|=k$.

Proof. One verifies that principal ideals for the $\leq_{k}$-order are described as follows:

$$
\downarrow u= \begin{cases}\{u\} & \text { if }|u|<k \\ p_{k-1}(u) A^{*} \cap A^{*} s_{k-1}(u) \cap \bigcap_{w \in F_{k}(u)} A^{*} w A^{*} & \text { otherwise }\end{cases}
$$

This shows that every positively $k$-testable language has the right form. It suffices now to verify that the languages $\{u\}, v A^{*}, A^{*} v$ and $A^{*} w A^{*}$, with $|u|<k-1,|v|=k-1$ and $|w|=k$, are order ideals for $\leq_{k}$. This is clear for $\{u\}$. Next, if $x \leq_{k} y$ and $y \in v A^{*}$, then $p_{k-1}(x)=p_{k-1}(y)=v$ and thus $x \in v A^{*}$. The proof for $A^{*} v$ is similar. Finally, if $x \leq_{k} y$ and $y \in A^{*} w A^{*}$, then $w \in F_{k}(y) \subseteq F_{k}(x)$ and thus $x \in A^{*} w A^{*}$.

In the sequel, we shall denote by $S(L)$ the syntactic ordered semigroup of a language $L$.

Proposition 4.18 Let $L$ be a language of $A^{+}$. For every $k \geq 0$, the following conditions are equivalent:

(1) $L$ is positively $k+1$-testable,

(2) $S(L) \in \mathbf{J}_{\mathbf{1}}^{+} * \mathbf{D}_{k}$,

(3) $S(L) \in \mathbf{J}_{1}^{+} * \mathbf{L} \mathbf{I}_{k}$,

Proof. Let $\mathcal{V}$ be the positive variety corresponding to $\mathbf{J}_{1}^{+} * \mathbf{D}_{k}$.

(1) implies (2). We show that $\mathcal{V}\left(A^{+}\right)$contains the positively $k+1$ testable languages. By Theorem 4.5, $\mathcal{V}\left(A^{+}\right)$contains the languages of the 
form $L, a A^{*}$ or $L a A^{*}$, where $L$ is suffix $k$-testable. It remains to show that $\mathcal{V}\left(A^{+}\right)$contains the languages of the form $v A^{*}$ and $A^{*} w A^{*}$, with $|v|=k$ and $|w|=k+1$. Let $v=v^{\prime} a$ and $w=w^{\prime} b$, with $a, b \in A$. If $v^{\prime}=1$, we already know that $a A^{*}$ is in $\mathcal{V}\left(A^{+}\right)$. Otherwise, $\left\{v^{\prime}\right\}$ is suffix $k$-testable and thus $v^{\prime} a A^{*}=v A^{*}$ belongs to $\mathcal{V}\left(A^{+}\right)$. Similarly, if $w^{\prime}=1$, we already know that $A^{*} b A^{*}$, which is recognized by $U_{1}^{+}$, is in $\mathcal{V}\left(A^{+}\right)$. Otherwise, $A^{*} w^{\prime}$ is suffix $k$-testable and thus $A^{*} w^{\prime} b A^{*}=A^{*} w A^{*}$ belongs to $\mathcal{V}\left(A^{+}\right)$.

(2) implies (3) is clear.

(3) implies (1). Theorem 4.5 shows that the languages of $\mathbf{J}_{\mathbf{1}}^{+} * \mathbf{L} \mathbf{I}_{k}$ are positive boolean combination of languages of the form $a A^{*}, L$ or $L a A^{*}$, where $a \in A$ and $L$ is prefix-suffix $k$-testable. By Proposition 4.16, $L$ is a finite union of languages of the form $\{u\}$ or $p A^{*} \cap A^{*} s$, with $|u|<k$ and $|p|=|s|=k$. Now $\{u\} a A^{*}$ and $\left(p A^{*} \cap A^{*} s\right) a A^{*}=p A^{*} \cap A^{*} s a A^{*}$ are positively $k+1$-testable.

A language is positively locally testable if it is positively $k$-testable for some $k$. Thus a positively locally testable language is a positive boolean combination of languages of the form $\{u\}, u A^{*}, A^{*} u$ or $A^{*} u A^{*}\left(u \in A^{+}\right)$. In particular, the languages of the form $F A^{*}, A^{*} G$ and $A^{*} H A^{*}$, for $F, G$ and $H$ finite, are positively locally testable.

Let $\mathbf{K}$ (resp. D) denote the variety of semigroups defined by the identity $x^{\omega} y=x^{\omega}$ (resp. $\left.y x^{\omega}=x^{\omega}\right)$. Finally, denote by LI the variety of semigroups defined by the identity $x^{\omega} y x^{\omega}=x^{\omega}$.

Corollary 4.19 Let $L$ be a language of $A^{+}$. The following conditions are equivalent:

(1) L is positively locally testable,

(2) $S(L) \in \mathbf{J}_{\mathbf{1}}^{+} * \mathbf{D}$,

(3) $S(L) \in \mathbf{J}_{1}^{+} * \mathbf{L I}$,

(4) $S(L) \in \mathbf{L} \mathbf{J}_{1}^{+}$

Proof. The equivalence of (2), (3) and (4) follows from [15, Corollary 4.4]. Furthermore, if $L$ is positively locally testable, then it is $k+1$-testable for some $k>0$, and by Proposition $4.18, S(L) \in \mathbf{J}_{\mathbf{1}}^{+} * \mathbf{L} \mathbf{I}_{k}$. This proves that (1) implies (3), since $\mathbf{L} \mathbf{I}_{k}$ is contained in LI. Finally, if $S(L) \in \mathbf{J}_{1}^{+} * \mathbf{L I}$, then by [17, Proposition 3.5], $S(L)$ divides a wreath product of the form $S \circ T$, with $S \in \mathbf{J}_{1}^{+}$and $T \in \mathbf{L I}$. Now $T \in \mathbf{L I}_{k}$ for some $k>0$, and $S(L)$ actually belongs to $\mathbf{J}_{1}^{+} * \mathbf{L} \mathbf{I}_{k}$. Applying Proposition 4.18, we conclude that (3) implies (1). 
Corollary 4.20 It is decidable whether a given rational language is positively locally testable.

\subsection{Varieties of the form $\mathrm{V} * \mathrm{LI}$}

In this section, we extend to varieties of ordered semigroups a result of Straubing [20] characterizing the languages corresponding to varieties of the form $\mathbf{V} * \mathbf{L I}$ and $\mathbf{V} * \mathbf{D}$.

Let $A$ be an alphabet. For each $k \geq 0$, let $C_{k}=A^{k}$. Then each word $u$ of length $k$ of $A^{*}$ defines a letter of $C_{k}$, denoted $[u]$ to avoid any confusion. Let $\sigma_{k}: A^{+} \rightarrow C_{k}^{*}$ be the function defined on $A^{k-1} A^{*}$ by

$$
\sigma_{k}\left(a_{1} a_{2} \cdots a_{n}\right)= \begin{cases}1 & \text { if } n=k-1 \\ {\left[a_{1} \cdots a_{k}\right]\left[a_{2} \cdots a_{k+1}\right] \cdots\left[a_{n-k+1} \cdots a_{n}\right]} & \text { if } n \geq k\end{cases}
$$

Thus $\sigma_{k}$ "spells" the factors of length $k$ of $u$.

Proposition 4.21 For every $k>0, \sigma_{k}$ is a subsequential function. It can be realized by a subsequential transducer whose transition semigroup belongs to $\mathbf{D}_{k-1}$.

Proof. A simple calculation shows that $\sigma_{k}$ is realized by the subsequential transducer $\mathcal{T}_{k}=\left(Q, A, C_{k}, \cdot, *, q_{0}, 1, \rho\right)$, where $Q$ is the set of words of length $<k, q_{0}$ is the empty word and the transition, output and final functions are defined on $Q$ by

$$
\begin{gathered}
q \cdot a= \begin{cases}q a & \text { if }|q|<k-1 \\
s_{k-1}(q a) & \text { if }|q|=k-1\end{cases} \\
q * a= \begin{cases}1 & \text { if }|q|<k-1 \\
{[q a]} & \text { if }|q|=k-1\end{cases} \\
\rho(q)= \begin{cases}1 & \text { if }|q|=k-1 \\
\text { undefined } & \text { otherwise }\end{cases}
\end{gathered}
$$

Now, the transition semigroup of $\mathcal{T}_{k}$ satisfies the identity $y x_{1} \cdots x_{k-1}=$ $x_{1} \cdots x_{k-1}$ and thus belongs to $\mathbf{D}_{k-1}$.

Example 4.1 If $A=\{a, b\}$, the transducer $\sigma_{3}$ is represented in Figure 4.2 below. 


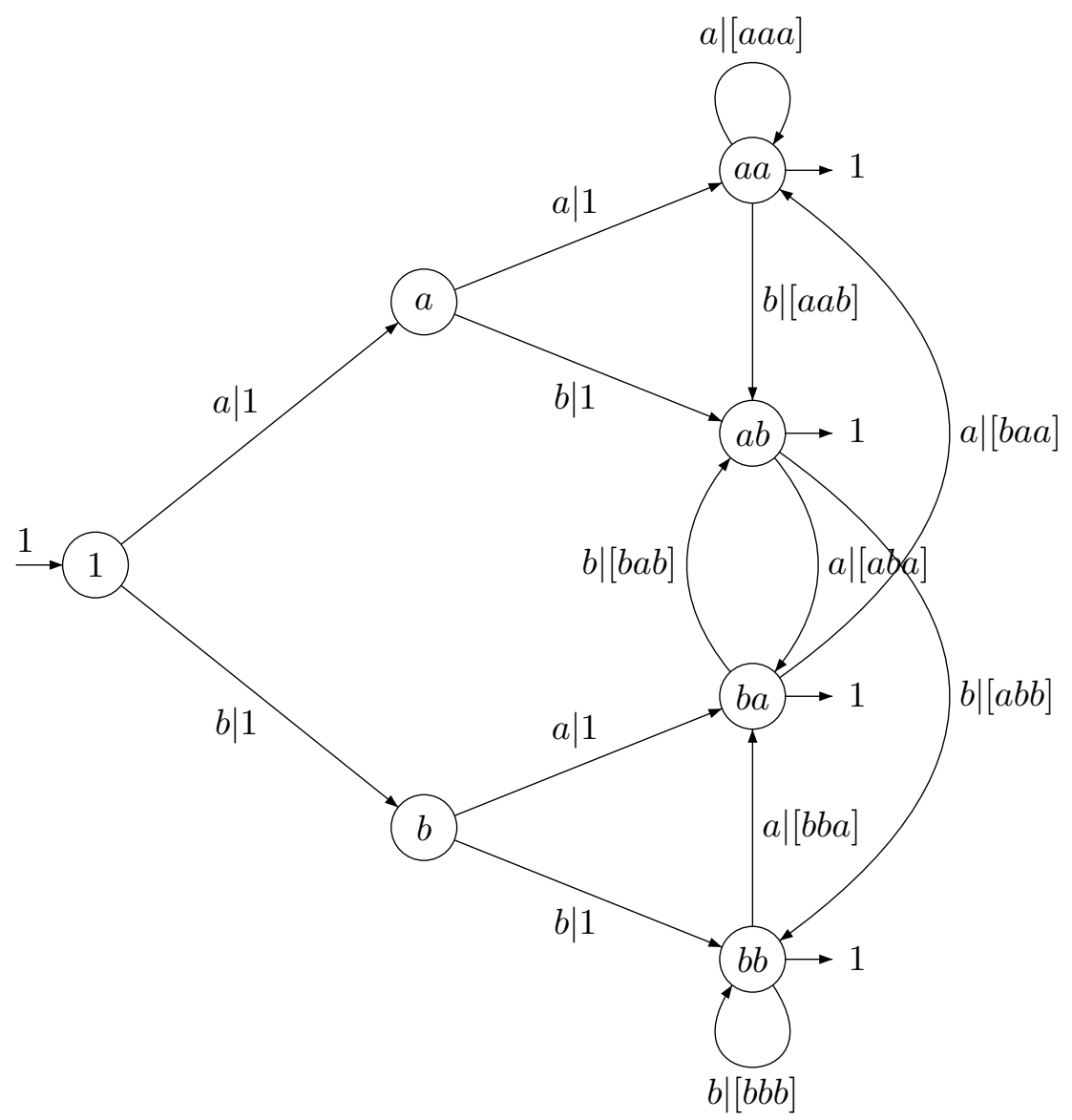

Figure 4.2: The transducer $\sigma_{3}$.

Theorem 4.22 Let $\mathbf{V}$ be a non-trivial variety of ordered monoids and let $\mathcal{V}$ be the corresponding positive variety. Then, for every language $L$ of $A^{+}$, the following conditions are equivalent:

(1) $L$ is a finite union of languages of the form $\{u\}$, with $|u|<k$ or $p A^{*} \cap \sigma_{k+1}^{-1}(K) \cap A^{*} s$ where $p, s \in A^{k}$ and $K \in \mathcal{V}\left(C_{k+1}^{*}\right)$,

(2) $L$ belongs to the smallest positive boolean algebra of $A^{+}$containing the prefix-suffix $k$-testable languages and the languages of the form $\sigma_{k+1}^{-1}(K)$ where $K \in \mathcal{V}\left(C_{k+1}^{*}\right)$,

(3) $S(L) \in \mathbf{V} * \mathbf{D}_{k}$,

(4) $S(L) \in \mathbf{V} * \mathbf{L I}_{k}$,

Proof. The equivalence of (3) and (4) follows from [15, Proposition 3.5].

(1) implies (2) is trivial.

(2) implies (4). Since $\mathbf{V} * \mathbf{L} \mathbf{I}_{k}$ is a variety of ordered semigroups containing $\mathbf{L} \mathbf{I}_{k}$, it suffices to show that if $L=\sigma_{k+1}^{-1}(K)$ with $K \in \mathcal{V}\left(C_{k+1}^{*}\right)$, then 
$S(L) \in \mathbf{V} * \mathbf{L I}_{k}$. But this follows from Theorem 3.2 and Proposition 4.21.

(3) implies (1). If $S(L) \in \mathbf{V} * \mathbf{D}_{k}$, then by Corollary 3.4, $L$ is a finite union of languages of the form $U \cap \sigma_{\varphi}^{-1}(V)$, where $U$ is suffix $k$-testable, $\varphi$ is a semigroup morphism from $A^{+}$into a semigroup $T$ of $\mathbf{D}_{k}$ and $V$ is a language of $\mathcal{V}\left(B_{T}^{*}\right)$.

Define a morphism $\alpha$ from $C_{k+1}^{+}$into $B_{T}^{*}$ by setting

$$
\alpha\left(\left[a_{1} \cdots a_{k+1}\right]\right)=\left(\varphi\left(a_{1} \cdots a_{k}\right), a_{k+1}\right)
$$

Since $T$ satisfies the identity $y x_{1} \cdots x_{k}=x_{1} \cdots x_{k}$, the following equalities hold, for each word $u=a_{1} \cdots a_{n}$.

$$
\begin{aligned}
\sigma_{\varphi}\left(a_{1} \cdots a_{n}\right) & =\left(1, a_{1}\right)\left(\varphi\left(a_{1}\right), a_{2}\right) \cdots\left(\varphi\left(a_{1} \cdots a_{n-1}\right), a_{n}\right) \\
& =\left(1, a_{1}\right) \cdots\left(\varphi\left(a_{1} \cdots a_{k-1}\right), a_{k}\right) \alpha\left(\left[a_{1} \cdots a_{k+1}\right]\right) \cdots \\
& =\sigma_{\varphi}\left(a_{1} \cdots a_{k}\right) \alpha\left(\sigma_{k+1}(u)\right)
\end{aligned}
$$

Therefore, if $|u| \geq k, \sigma_{\varphi}(u) \in V$ if and only if

$$
\alpha\left(\sigma_{k+1}(u)\right) \in w^{-1} V \text { where } w=\sigma_{\varphi}\left(p_{k}(u)\right)
$$

In order to handle the set $W=\sigma_{\varphi}^{-1}(V)$ more easily, it is convenient to split it into several pieces. Denoting by $F$ the set of words of $W$ of length $<k$, we have

$$
W=F \cup\left(\bigcup_{p \in A^{k}}\left(p A^{*} \cap W\right)\right)
$$

Now $F$ is a finite set and in view of condition (4)

$$
p A^{*} \cap W=p A^{*} \cap \sigma_{k+1}^{-1}\left(\alpha^{-1}\left(\sigma_{\varphi}(p)^{-1} V\right)\right)
$$

Now, $V \in \mathcal{V}\left(B_{T}^{*}\right)$, and since a positive variety is closed under residuals and under inverse of morphisms, the language $\sigma_{\varphi}(p)^{-1} V$ is in $\mathcal{V}\left(B_{T}^{*}\right)$ and $\alpha^{-1}\left(\sigma_{\varphi}(p)^{-1} V\right)$ is in $\mathcal{V}\left(C_{k+1}^{*}\right)$.

Finally, since $U$ is suffix $k$-testable, it is a finite union of languages of the form $\{u\}$, with $|u|<k$ or $A^{*} s$, with $s \in A^{k}$. It follows that $U \cap \sigma_{\varphi}^{-1}(V)$ is a finite union of the form $\{u\}$, with $|u|<k$ and of languages of the form

$$
p A^{*} \cap \sigma_{k+1}^{-1}(K) \cap A^{*} s
$$

where $|p|=|s|=k$ and $K \in \mathcal{V}\left(C_{k+1}^{*}\right)$. Thus $L$ satisfies (1). 
Corollary 4.23 Let $\mathbf{V}$ be a non-trivial variety of ordered monoids and let $\mathcal{V}$ be the corresponding positive variety. Then, for every language $L$ of $A^{+}$, the following conditions are equivalent:

(1) $L$ is a finite union of languages of the form $\{u\}$, with $u \in A^{+}$or $p A^{*} \cap \sigma_{k}^{-1}(K) \cap A^{*} s$ where $p, s \in A^{k-1}$ and $K \in \mathcal{V}\left(C_{k}^{*}\right)$ for some $k>0$,

(2) L belongs to the smallest positive boolean algebra of $A^{+}$containing the prefix-suffix testable languages and the languages of the form $\sigma_{k}^{-1}(K)$ where $K \in \mathcal{V}\left(C_{k}^{*}\right)$ for some $k \geq 0$,

(3) $S(L) \in \mathbf{V} * \mathbf{D}$,

(4) $S(L) \in \mathbf{V} * \mathbf{L I}$,

\section{Concatenation hierarchies}

We first recall the definition of polynomial closure of a class of languages, as defined in [16]. There are actually two slightly different notions of polynomial closure, one for + -classes and one for $*$-classes.

The polynomial closure of a class of languages $\mathcal{L}$ of $A^{+}$is the set of languages of $A^{+}$that are finite unions of languages of the form $u_{0} L_{1} u_{1} \cdots L_{n} u_{n}$, where $n \geq 0$, the $u_{i}$ 's are words of $A^{*}$ and the $L_{i}$ 's are elements of $\mathcal{L}$. If $n=0$, one requires of course that $u_{0}$ is not the empty word.

The polynomial closure of a class of languages $\mathcal{L}$ of $A^{*}$ is the set of languages that are finite unions of languages of the form $L_{0} a_{1} L_{1} \cdots a_{n} L_{n}$, where the $a_{i}$ 's are letters and the $L_{i}$ 's are elements of $\mathcal{L}$.

Concatenation hierarchies are now obtained by alternating the use of the polynomial closure and of the boolean closure. More precisely, if $\mathcal{V}$ is a variety of languages, the concatenation hierarchy of basis $\mathcal{V}$ is the hierarchy of classes of languages defined as follows:

(1) Level 0 is $\mathcal{V}$.

(2) For every integer $n \geq 0$, level $n+1 / 2$ is the polynomial closure of level $n$.

(3) For every integer $n \geq 0$, level $n+1$ is the boolean closure of level $n+1 / 2$.

It is known that every full level is a variety of languages and that every half level is a positive variety of languages $[14,16]$.

The Straubing-Thérien hierarchy is the hierarchy of positive varieties of languages based on the trivial $*$-variety. The variety (resp. positive variety) corresponding to the level $n$ (resp. $n+1 / 2$ ) of the hierarchy is denoted by 
$\mathbf{V}_{n}$ (resp. $\mathbf{V}_{n+1 / 2}$ ). In particular, it is known that $\mathbf{V}_{1 / 2}=\mathbf{J}^{+}, \mathbf{V}_{1}=\mathbf{J}$ [18], and an effective characterization of $\mathbf{V}_{3 / 2}$ is given in [16]. No such characterization is known for the upper levels. See $[14,16]$ for more details.

The dot-depth hierarchy is the hierarchy of positive varieties of languages based on the trivial +-variety. The variety (resp. positive variety) corresponding to the level $n$ (resp. $n+1 / 2$ ) of the hierarchy is denoted by $\mathbf{B}_{n}$ (resp. $\mathbf{B}_{n+1 / 2}$ ). In particular, it is known that $\mathbf{B}_{1 / 2}=\mathbf{L} \mathbf{J}^{+}$, and an effective characterization of $\mathbf{B}_{1}$ is given in [8,9].

An important result of Straubing [20] states that the two hierarchies are related as follows. For every integer $n \geq 0$,

$$
\mathbf{B}_{n}=\mathbf{V}_{n} * \mathbf{L I} \text {. }
$$

The main result of this section shows that this relation holds for the half levels as well. As we will see, it implies that a level (resp. a half level) of the dot-depth hierarchy is decidable if and only if the corresponding level of the Straubing-Thérien hierarchy is decidable.

As an appetizer, we first study the languages of dot-depth $1 / 2$.

\subsection{Languages of dot-depth $1 / 2$}

It is easy to see that the languages of dot-depth $1 / 2$ are the finite unions of languages of the form

$$
u_{0} A^{*} u_{1} A^{*} \cdots u_{k-1} A^{*} u_{k}
$$

where $k \geq 0, u_{0}, \ldots, u_{k} \in A^{*}$ and $u_{0} u_{1} \cdots u_{k} \neq 1$. It is shown in [16], as a corollary of a more general result, that a language of $A^{+}$is of dot-depth $1 / 2$ if and only if its ordered syntactic semigroup belongs to the variety $\mathbf{L} \mathbf{J}^{+}$, defined by the identity $x^{\omega} y x^{\omega} \leq x^{\omega}$. This result will be recovered in a direct fashion, and supplemented by a detailed study of the languages of dot-depth $1 / 2$.

For each sequence $u_{1}, \ldots, u_{n}$ of words of equal length of $A^{+}$, set $L\left(u_{1}, \ldots, u_{n}\right)=\left\{u \in A^{+} \mid u_{1}, \ldots, u_{n}\right.$ occur in this order as factors of $\left.u\right\}$ For instance $L(u)=A^{*} u A^{*}$, and $L(a b, b a)=A^{*} a b A^{*} b a A^{*} \cup A^{*} a b a A^{*}$.

Theorem 4.22 and Proposition 4.8 are both relevant to study the languages corresponding to the variety $\mathbf{J}^{+} * \mathbf{D}_{k}$. Both are actually used to obtain the next result.

Theorem 5.1 Let $k>0$ and let $L$ be a language of $A^{+}$. The following conditions are equivalent: 
(1) $L$ is a finite union of languages of the form $\{u\}$, with $|u|<k$ or $p A^{*} \cap L\left(u_{1}, \ldots, u_{n}\right) \cap s A^{*}$, where $p, s \in A^{k}$ and $u_{1}, \ldots, u_{n}$ is a sequence of words of $A^{k+1}$.

(2) $L$ belongs to the smallest positive boolean algebra containing the languages of the form $\{u\}$, with $0<|u|<k$, a $A^{*}$, for $a \in A$ and $A^{*} s$, with $|s|=k$, which is also closed under the operation $L \rightarrow L a A^{*}$.

(3) $S(L) \in \mathbf{J}^{+} * \mathbf{D}_{k}$.

(4) $S(L) \in \mathbf{J}^{+} * \mathbf{L} \mathbf{I}_{k}$.

Proof. The equivalence of (3) and (4) follows from [15, Proposition 3.8].

The equivalence of (1) and (3) is a consequence of Theorem 4.22, applied to $\mathbf{V}=\mathbf{J}^{+}$. Indeed, we know from [13, Theorem 6.4] (see also [14]) that the languages $K$ of $C_{k+1}^{*}$ recognized by an ordered monoid of $\mathbf{J}^{+}$are finite unions of languages of the form $C_{k+1}^{*} c_{1} C_{k+1}^{*} c_{2} \cdots C_{k+1}^{*} c_{n} C_{k+1}^{*}$, with $c_{1}, \ldots, c_{n} \in$ $C_{k+1}$. Therefore, the languages of the form $\sigma_{k+1}^{-1}(K)$, with $K$ recognized by an ordered monoid of $\mathbf{J}^{+}$, are exactly the finite unions of languages of the form $L\left(u_{1}, \ldots, u_{n}\right)$, with $u_{1}, \ldots, u_{n} \in A^{k+1}$ and the languages of the form $p A^{*} \cap \sigma_{k+1}^{-1}(K) \cap A^{*} s$ are exactly the finite unions of languages of the form $p A^{*} \cap L\left(u_{1}, \ldots, u_{n}\right) \cap A^{*} s$.

The equivalence of (2) and (3) follows from Proposition 4.8.

If $k>1$, it is actually not necessary to include the languages of the form $a A^{*}$ in condition (2). It suffices to know that $\mathcal{C}\left(A^{+}\right)$contains the languages of the form $\{a\}$ for $a \in A$, and that $\mathcal{C}\left(A^{+}\right)$is closed under the operation $L \rightarrow L a A^{*}$ since

$$
a A^{*}=\{a\} \cup \bigcup_{b \in A}\{a\} b A^{*}
$$

Corollary 5.2 Let $L$ be a language of $A^{+}$. The following conditions are equivalent.

(1) $L$ is of dot-depth $1 / 2$,

(2) $L$ is a finite union of languages of the form $\{u\}$, with $u \in A^{+}$or $p A^{*} \cap L\left(u_{1}, \ldots, u_{n}\right) \cap A^{*} s$, where, for some $k>0, p, s \in A^{k-1}$ and $u_{1}$, $\ldots, u_{n}$ is a sequence of words of $A^{k}$.

(3) L belongs to the smallest positive boolean algebra containing the suffixtestable languages which is closed under the operation $L \rightarrow L a A^{*}$,

(4) $S(L) \in \mathbf{J}^{+} * \mathbf{D}$,

(5) $S(L) \in \mathbf{J}^{+} * \mathbf{L I}$,

(6) $S(L) \in \mathbf{L J}^{+}$. 
Proof. The equivalence of (2), (3), (4) and (5) follows immediately from Theorem 5.1 and the equivalence of (5) and (6) follows from [15, Corollary 4.10].

There are many ways to show that a language $L$ of dot-depth $1 / 2$ satisfies one of the equivalent conditions (2)-(6). An elementary way is to verify directly that $S(L)$ belongs to $\mathbf{L} \mathbf{J}^{+}$, a result which is actually a special case of [16, Corollary 5.3]. Coming back to the definition of the syntactic order, it amounts to showing that $L$ satisfies the following pumping condition: if, for some words $u, x$, and $v$ of $A^{+}, u x^{+} v \subseteq L$ then, for each word $y \in A^{+}$, $u x^{+} y x^{+} v \subseteq L$. But this condition is trivially satisfied by a language of the form $u_{0} A^{*} u_{1} A^{*} \cdots u_{r-1} A^{*} u_{r}$.

For the proof that (2) implies (1), we essentially reproduce the informal argument used in [20, p. 88]. It suffices to show that if $L=$ $p A^{*} \cap L\left(u_{1}, \ldots, u_{n}\right) \cap A^{*} s$ with $k>0, p, s \in A^{k-1}$ and $u_{1}, \ldots, u_{n} \in A^{k}$, then $L$ is of dot-depth $1 / 2$. In any word $u$ of $L, p$ appears as a prefix, $s$ has a suffix, and the words $u_{1}, \ldots, u_{n}$ appear in sequence as factors of $u$, possibly with some overlap. One can coalesce the overlapping factors into a sequence of non-overlapping segments $v_{0}, v_{1}, \ldots, v_{r}$ in such a way that $u \in v_{0} A^{*} v_{1} A^{*} \cdots v_{r-1} A^{*} v_{r}$. For instance, if $p=a b, u_{1}=a b b, u_{2}=b a b$, $u_{3}=b a a$ and $s=a b$, one may take $v_{0}=a b b, v_{1}=b a b a a$ and $v_{2}=a b$, or $v_{0}=a b b a b$ and $v_{1}=b a a b$ or even just $v_{0}=a b b a b a a b$ (there are other possibilities). Let us say in this case that the sequence $\left(v_{0}, v_{1}, \ldots, v_{r}\right)$ is adapted to $L$. Since the sum of the length of the $v_{i}$ 's is bounded by $\left|p u_{1} \ldots u_{n} s\right|$, there are only finitely many such sequences. Now $L$ is the union of the languages of the form $v_{0} A^{*} v_{1} A^{*} \cdots v_{r-1} A^{*} v_{r}$, where $\left(v_{0}, v_{1}, \ldots, v_{r}\right)$ runs over the finite set of sequences adapted to $L$. Thus $L$ is of dot-depth $1 / 2$.

\subsection{An extension of Straubing's result}

In this section, we extend Straubing's result on concatenation hierarchies by showing it still holds for the half levels. Our proof is inspired by Straubing's original proof but we introduce a few simplifications. Furthermore, the proof is now entirely self-contained. In the sequel, we use the term half-integer to denote a rational number of the form $n$ or $n+1 / 2$ for some non-negative integer $n$.

Theorem 5.3 For every half-integer $n>0$, the formula $\mathbf{B}_{n}=\mathbf{V}_{n} * \mathbf{L I}$ holds. 
Proof. Denote by $\mathcal{B}_{n}, \mathcal{V}_{n}$ and $\mathcal{W}_{n}$ the positive varieties of languages corresponding respectively to $\mathbf{B}_{n}, \mathbf{V}_{n}$ and $\mathbf{V}_{n} * \mathbf{L I}$. By the variety theorem [5, 13], it suffices to establish the equality $\mathcal{B}_{n}=\mathcal{W}_{n}$. This is done by induction.

For $n=1 / 2$, the formula $\mathbf{B}_{1 / 2}=\mathbf{V}_{1 / 2} * \mathbf{L I}$ follows directly from Corollary 5.2 , since $\mathbf{V}_{1 / 2}=\mathbf{J}^{+}$. There are now two distinct inductions steps: for an integer $n$, passing from level $n+1 / 2$ to level $n+1$ and passing from level $n$ to level $n+1 / 2$. The first one is by far the easier one, since it only relies on the fact that boolean operations commute with inverses of functions.

Lemma 5.4 Let $n$ be an integer. If $\mathbf{B}_{n+1 / 2}=\mathbf{V}_{n+1 / 2} * \mathbf{L I}$, then $\mathbf{B}_{n+1}=$ $\mathbf{V}_{n+1} * \mathbf{L I}$.

Proof. We first establish the inclusion $\mathcal{W}_{n+1}\left(A^{+}\right) \subseteq \mathcal{B}_{n+1}\left(A^{+}\right)$. By Corollary 4.23, every language $L$ of $\mathcal{W}_{n+1}\left(A^{+}\right)$is a positive boolean combination of prefix-suffix testable languages and of languages of the form $\sigma_{k}^{-1}(K)$, where $K \in \mathcal{V}_{n+1}\left(C_{k}^{*}\right)$ for some $k>0$. Now, by the definition of $\mathcal{V}_{n+1}, K$ itself is a boolean combination of languages of $\mathcal{V}_{n+1 / 2}\left(C_{k}^{*}\right)$. Since boolean operations commute with $\sigma_{k}^{-1}, L$ is a boolean combination of prefix-suffix testable languages and of languages of the form $\sigma_{k}^{-1}(R)$, where $R \in \mathcal{V}_{n+1 / 2}\left(C_{k}^{*}\right)$ for some $k>0$. Now, by Corollary 4.23 again, such a language $\sigma_{k}^{-1}(R)$ belongs to $\mathcal{W}_{n+1 / 2}\left(A^{+}\right)$, and thus, by the induction hypothesis, to $\mathcal{B}_{n+1 / 2}\left(A^{+}\right)$. It follows that $L$ belongs to $\mathcal{B}_{n+1}\left(A^{+}\right)$.

The opposite inclusion $\mathcal{B}_{n+1}\left(A^{+}\right) \subseteq \mathcal{W}_{n+1}\left(A^{+}\right)$is proved in a similar way. By definition, a language $L$ of $\mathcal{B}_{n+1}\left(A^{+}\right)$is a boolean combination of a family $\left(L_{i}\right)_{1 \leq i \leq r}$ of languages of $\mathcal{B}_{n+1 / 2}\left(A^{+}\right)$which are also, by the induction hypothesis, in $\mathcal{W}_{n+1 / 2}\left(A^{+}\right)$. It follows that $S\left(L_{i}\right) \in \mathbf{V}_{n+1 / 2} * \mathbf{L I}$ and thus $S\left(L_{i}\right) \in \mathbf{V}_{n+1 / 2} * \mathbf{L} \mathbf{I}_{k_{i}}$ for some $k_{i}>0$. Let $k=1+\max _{1 \leq i \leq r} k_{i}$. Then $S\left(L_{i}\right) \in \mathbf{V}_{n+1 / 2} * \mathbf{L} \mathbf{I}_{k-1}$ for each $i$, and by Theorem 4.22, each $L_{i}$ is a positive boolean combination of prefix-suffix testable languages and of languages of the form $\sigma_{k}^{-1}(R)$, where $R \in \mathcal{V}_{n+1 / 2}\left(C_{k}^{*}\right)$. Since boolean operations commute with $\sigma_{k}^{-1}$, any boolean combination of languages of the form $\sigma_{k}^{-1}(R)$, where $R \in \mathcal{V}_{n+1 / 2}\left(C_{k}^{*}\right)$ can be written as $\sigma_{k}^{-1}(K)$ with $K \in \mathcal{V}_{n+1}\left(C_{k}^{*}\right)$. It follows that $L$ is a boolean combination of languages of prefix-suffix testable languages and of languages of the form $\sigma_{k}^{-1}(K)$, with $K \in \mathcal{V}_{n+1}\left(C_{k}^{*}\right)$. Now, each of these languages is in $\mathcal{W}_{n+1}\left(A^{+}\right)$, and since $\mathcal{W}_{n+1}$ is a variety, $\mathcal{W}_{n+1}\left(A^{+}\right)$is closed under boolean operations. Therefore $L \in \mathcal{W}_{n+1}\left(A^{+}\right)$.

Before we proceed with the second induction step, we note the following formula, an immediate consequence of the definition of $\sigma_{k}$. If $x$ and $y$ are 
words, with $x$ and $y$ of length $\geq k-1$, then

$$
\sigma_{k}(x y)=\sigma_{k}(x) \sigma_{k}\left(s_{k-1}(x) y\right)
$$

The second induction step relies on a lemma which requires a careful analysis of overlapping factors.

Lemma 5.5 (Inversion formula) For $0 \leq i \leq r$, let $u_{i}$ be a word of $A^{*}$ of length $\geq k-1$ and let $p_{i}=p_{k-1}\left(u_{i}\right), s_{i+1}=s_{k-1}\left(u_{i}\right)$ and $u_{i}=p_{i} u_{i}^{\prime}$. Let, for $1 \leq i \leq r, K_{i}$ be a language of $C_{k}^{*}$, and let

$$
H_{i}=\left\{x \in A^{*} \mid \sigma_{k}\left(s_{i} x\right) \in K_{i} \text { and } s_{k-1}\left(s_{i} x\right)=p_{i}\right\}
$$

Then the following equality holds:

$$
\begin{aligned}
u_{0} H_{1} u_{1}^{\prime} \cdots H_{r} u_{r}^{\prime} & = \\
p_{0} A^{*} & \cap \sigma_{k}^{-1}\left[\sigma_{k}\left(u_{0}\right) K_{1} \sigma_{k}\left(u_{1}\right) \cdots \sigma_{k}\left(u_{r-1}\right) K_{r} \sigma_{k}\left(u_{r}\right)\right] \cap A^{*} s_{r+1}
\end{aligned}
$$

Proof. Denote respectively by $L$ and $R$ the left and right hand sides of the formula. If $u \in L, u$ can be written as $u=u_{0} v_{1} u_{1}^{\prime} \cdots v_{r} u_{r}^{\prime}$ where, for $1 \leq i \leq r, v_{i} \in H_{i}$. We first prove by induction on $i$ that, for $0 \leq i \leq r$,

$$
s_{k-1}\left(u_{0} v_{1} u_{1}^{\prime} \cdots v_{i} u_{i}^{\prime}\right)=s_{i+1}
$$

The result is trivial if $i=0$. Assuming that the formula holds for $i-1$, we have $s_{k-1}\left(u_{0} v_{1} u_{1}^{\prime} \cdots v_{i} u_{i}^{\prime}\right)=s_{k-1}\left(s_{i} v_{i} u_{i}^{\prime}\right)$. Now since $v_{i} \in H_{i}, s_{k-1}\left(s_{i} v_{i}\right)=$ $p_{i}$. Therefore, $s_{k-1}\left(s_{i} v_{i} u_{i}^{\prime}\right)=s_{k-1}\left(p_{i} u_{i}^{\prime}\right)=s_{k-1}\left(u_{i}\right)=s_{i+1}$.

It follows in particular that $u \in p_{0} A^{*} \cap A^{*} s_{r+1}$. We now prove by induction on $i$ that, for $0 \leq i \leq r$,

$$
\sigma_{k}\left(u_{0} v_{1} u_{1}^{\prime} \cdots v_{i} u_{i}^{\prime}\right)=\sigma_{k}\left(u_{0}\right) \sigma_{k}\left(s_{1} v_{1}\right) \sigma_{k}\left(u_{1}\right) \cdots \sigma_{k}\left(s_{i} v_{i}\right) \sigma_{k}\left(u_{i}\right)
$$

For instance, if $k=4, u_{0}=a b c a, v_{1}=c, u_{1}=c a c b, v_{2}=b, u_{2}=c b b a$, then $u_{1}^{\prime}=b, u_{2}^{\prime}=a, u=a b c a c b b a$ and $\sigma(u)=[a b c a][b c a c][c a c b][a c b b][c b b a]$.

The result is obvious if $i=0$. Assume that the formula holds for $i-1$. Setting $x=u_{0} v_{1} u_{1}^{\prime} \cdots v_{i-1} u_{i-1}^{\prime}$, we have $s_{k-1}(x)=s_{i}$ by (6). It follows by (5) that $\sigma_{k}\left(u_{0} v_{1} u_{1}^{\prime} \cdots v_{i} u_{i}^{\prime}\right)=\sigma_{k}(x) \sigma_{k}\left(s_{i} v_{i} u_{i}^{\prime}\right)$ and, since $s_{k-1}\left(s_{i} v_{i}\right)=p_{i}$,

$$
\sigma_{k}\left(s_{i} v_{i} u_{i}^{\prime}\right)=\sigma_{k}\left(s_{i} v_{i}\right) \sigma_{k}\left(s_{k-1}\left(s_{i} v_{i}\right) u_{i}^{\prime}\right)=\sigma_{k}\left(s_{i} v_{i}\right) \sigma_{k}\left(p_{i} u_{i}^{\prime}\right)=\sigma_{k}\left(s_{i} v_{i}\right) \sigma_{k}\left(u_{i}\right)
$$

which completes the proof of (7) by induction. It follows from (7) that $\sigma_{k}(u) \in \sigma_{k}\left(u_{0}\right) K_{1} \sigma_{k}\left(u_{1}\right) K_{2} \cdots K_{r} \sigma_{k}\left(u_{r}\right)$ and thus $u \in R$. Therefore $L \subseteq R$. 
To establish the opposite inclusion, consider a word $u \in R$. Then $u \in$ $p_{0} A^{*} \cap A^{*} s_{r+1}$ and $u \in \sigma_{k}^{-1}\left[\sigma_{k}\left(u_{0}\right) x_{1} \sigma_{k}\left(u_{1}\right) \cdots \sigma_{k}\left(u_{r-1}\right) x_{r} \sigma_{k}\left(u_{r}\right)\right]$ for some $x_{1} \in K_{1}, \ldots, x_{r} \in K_{r}$. Note that each of the words $x_{i}$ is necessarily of the form $\left[a_{1} \cdots a_{k}\right]\left[a_{2} \cdots a_{k+1}\right] \cdots\left[a_{r_{i}} \cdots a_{k+r_{i}-1}\right]$, where $a_{1} \cdots a_{k-1}$ is equal to $s_{i}$ and $a_{r_{i}+1} \cdots a_{k+r_{i}-1}$ is equal to $p_{i}$. It may happen that $x_{i}=1$, and $s_{i}=p_{i}$.

Let $\pi_{k}: C_{k}^{*} \rightarrow A^{*}$ be the morphism defined by $\pi_{k}\left(\left[a_{1} \cdots a_{k}\right]\right)=a_{k}$. Thus, in particular, $\pi_{k}\left(x_{i}\right)=a_{k} \cdots a_{k+r_{i}-1}$. Let, for $1 \leq i \leq r, v_{i}=\pi_{k}\left(x_{i}\right)$. For instance, if $k=4, u_{0}=a b c a b, x_{1}=[c a b a], u_{1}=a b a b, x_{2}=1, u_{2}=b a b c$, $x_{3}=[a b c c][b c c a], u_{3}=c c a b$, and

$$
\sigma_{k}(u)=[a b c a][b c a b][c a b a][a b a b][b a b c][a b c c][b c c a][c c a b]
$$

then $v_{1}=a, v_{2}=1$ and $v_{3}=c a$.

We have $\sigma_{k}\left(s_{i} v_{i}\right)=\sigma_{k}\left(s_{i} \pi_{k}\left(x_{i}\right)\right)=x_{i} \in K_{i}$ and

$$
s_{k-1}\left(s_{i} v_{i}\right)=s_{k-1}\left(s_{i} \pi_{k}\left(x_{i}\right)\right)=s_{k-1}\left(a_{1} \cdots a_{k+r_{i}-1}\right)=p_{i} .
$$

Let $w=u_{0} v_{1} u_{1}^{\prime} \cdots v_{r} u_{r}^{\prime}$. Since $v_{i} \in H_{i}$ by construction, $w \in L$. Thus, by (7),

$$
\sigma(w)=\sigma_{k}\left(u_{1}\right) x_{1} \cdots x_{r} \sigma_{k}\left(u_{r}\right)=\sigma(u) .
$$

Since $\sigma_{k}$ is one-to-one on $A^{k} A^{*}$, it follows $u=w$ and $u \in L$. Therefore $L=R$.

We are now ready for the proof of the second induction step.

Lemma 5.6 Let $n$ be an integer. If $\mathbf{B}_{n}=\mathbf{V}_{n} * \mathbf{L I}$, then $\mathbf{B}_{n+1 / 2}=\mathbf{V}_{n+1 / 2} *$ LI.

Proof. We first establish the inclusion $\mathcal{W}_{n+1 / 2}\left(A^{+}\right) \subseteq \mathcal{B}_{n+1 / 2}\left(A^{+}\right)$. By Corollary 4.23, every language of $\mathcal{W}_{n+1 / 2}\left(A^{+}\right)$is a finite union of a finite language and of languages of the form $p_{0} A^{*} \cap \sigma_{k}^{-1}(K) \cap A^{*} s_{r+1}$, where $K \in$ $\mathcal{V}_{n+1 / 2}\left(C_{k}^{*}\right)$ for some $k>0$ and $p_{0}, s_{r+1} \in A^{k-1}$. Since $K$ is a finite union of languages of the form $K_{1} c_{1} K_{2} \cdots c_{r-1} K_{r}$, where the $K_{i}$ 's are languages of $\mathcal{V}_{n}\left(C_{k}^{*}\right)$ and the $c_{i}$ 's are letters of $C_{k}$, it suffices to consider a language of the form

$$
L=p_{0} A^{*} \cap \sigma_{k}^{-1}\left(K_{1} c_{1} K_{2} \cdots c_{r-1} K_{r}\right) \cap A^{*} s_{r+1}
$$

Setting $u_{0}=p_{0}, u_{r}=s_{r+1}$ and, for $1 \leq i \leq r-1, c_{i}=\left[u_{i}\right]$, we have $\sigma_{k}\left(u_{0}\right)=1, \sigma_{k}\left(u_{r}\right)=1$ and, for $1 \leq i \leq r-1, \sigma_{k}\left(u_{i}\right)=c_{i}$. Therefore

$$
L=p_{0} A^{*} \cap \sigma_{k}^{-1}\left[\sigma_{k}\left(u_{0}\right) K_{1} \sigma_{k}\left(u_{1}\right) K_{2} \cdots \sigma_{k}\left(u_{r-1}\right) K_{r} \sigma_{k}\left(u_{r}\right)\right] \cap A^{*} s_{r+1}
$$


Setting, as in Lemma 5.5,

$$
H_{i}=\left\{x \in A^{*} \mid \sigma_{k}\left(s_{i} x\right) \in K_{i} \text { and } s_{k-1}\left(s_{i} x\right)=p_{i}\right\}
$$

we have, by the inversion formula,

$$
L=u_{0} H_{1} u_{1}^{\prime} \cdots H_{r} u_{r}^{\prime}
$$

Now, by Corollary 4.23 , each of the languages $\sigma_{k}^{-1}\left(K_{i}\right)$ belongs to $\mathcal{W}_{n}\left(A^{+}\right)$ and thus to $\mathcal{B}_{n}\left(A^{+}\right)$by the induction hypothesis. Observing that

$$
H_{i}=s_{i}^{-1}\left(\sigma_{k}^{-1}\left(K_{i}\right) \cap A^{*} p_{i}\right)
$$

it follows, since a positive variety is closed under intersection and under residuals, that $H_{i}$ is also in $\mathcal{B}_{n}\left(A^{+}\right)$. Therefore $L$ belongs to $\mathcal{B}_{n+1 / 2}\left(A^{+}\right)$.

For the opposite inclusion, consider a language of $\mathcal{B}_{n+1 / 2}\left(A^{+}\right)$. By definition, it is a finite union of languages of the form

$$
L=x_{0} L_{1} x_{1} \cdots L_{r} x_{r}
$$

where the $x_{i}$ 's are words of $A^{*}$ and the $L_{i}$ 's are languages of $\mathcal{B}_{n}\left(A^{+}\right)$. By the induction hypothesis, $\mathcal{B}_{n}\left(A^{+}\right)=\mathcal{W}_{n}\left(A^{+}\right)$and thus, all the ordered semigroups $S\left(L_{i}\right)$ are in $\mathbf{V} * \mathbf{L I}$. It follows, by Corollary 4.23 , that for some $k>0$, each $L_{i}$ is the union of a finite language and of languages of the form

$$
L_{i}=s_{i} A^{*} \cap \sigma_{k}^{-1}\left(K_{i}\right) \cap A^{*} p_{i}
$$

where $K_{i} \in \mathcal{V}_{n}\left(C_{k}^{*}\right)$, and $p_{i}, s_{i}$ are words of length $k-1$. By using the distributivity of product over union, we may assume that $L$ is of the form (8), where each $L_{i}$ is of the form (9). Setting $u_{0}=x_{0} s_{1}, u_{r}^{\prime}=x_{r}, u_{r}=p_{r} u_{r}^{\prime}$ and, for $1 \leq i \leq r-1, u_{i}^{\prime}=x_{i} s_{i+1}, u_{i}=p_{i} u_{i}^{\prime}$ and $H_{i}=s_{i}^{-1} L_{i}$, we have

$$
L=u_{0} H_{1} u_{1}^{\prime} \cdots H_{r} u_{r}^{\prime}
$$

Furthermore,

$$
H_{i}=\left\{x \in A^{*} \mid s_{i} x \in L_{i}\right\}=\left\{x \in A^{*} \mid \sigma_{k}\left(s_{i} x\right) \in K_{i} \text { and } s_{k-1}\left(s_{i} x\right)=p_{i}\right\}
$$

Therefore, Lemma 5.5 can be applied to obtain

$$
L=p_{0} A^{*} \cap \sigma_{k}^{-1}\left[\sigma_{k}\left(u_{0}\right) K_{1} \sigma_{k}\left(u_{1}\right) \cdots \sigma_{k}\left(u_{r-1}\right) K_{r} \sigma_{k}\left(u_{r}\right)\right] \cap A^{*} s_{r+1}
$$

where $p_{0}=p_{k-1}\left(u_{0}\right)$ and $s_{r+1}=s_{k-1}\left(u_{r}\right)$. It follows that $L$ belongs to $\mathcal{W}_{n+1 / 2}\left(A^{+}\right)$and this completes the proof of Lemma 5.6.

The induction is now complete and Theorem 5.3 is proved. 
Corollary 5.7 For every half-integer $n$, the variety $\mathbf{B}_{n}$ is decidable if and only if $\mathbf{V}_{n}$ is decidable.

Proof. The varieties $\mathbf{V}_{0}=\mathbf{I}, \mathbf{V}_{1 / 2}=\mathbf{J}^{+}$and $\mathbf{V}_{1}=\mathbf{J}$ are all decidable and so are the varieties $\mathbf{B}_{0}=\mathbf{L I}, \mathbf{B}_{1 / 2}=\mathbf{L} \mathbf{J}^{+}$and $\mathbf{B}_{1}$. Furthermore, $B_{2}^{+}$belongs to $\mathbf{V}_{3 / 2}$ and by [15, Corollary 3.7], if $\mathbf{V}_{n}$ is decidable for $n \geq 3 / 2$, then $\mathbf{B}_{n}$ is. The converse implication follows from the observation that $\mathbf{V}_{n}$ is the class of ordered monoids in $\mathbf{B}_{n}$. This observation follows immediately from the definition of the semidirect product of ordered semigroups and from the well known fact that $\mathbf{V} * \mathbf{L I}$ is contained in $\mathbf{L V}$.

In particular, since $\mathbf{V}_{3 / 2}$ is decidable [16], Corollary 5.7 shows that $\mathbf{B}_{3 / 2}$ is decidable, a result first proved by Glaßer and Schmitz [7] using different techniques.

\section{Acknowledgements}

The authors would like to thank Ben Steinberg and the anonymous referee for their many suggestions, which led to a significant improvement of the paper.

\section{References}

[1] J. Almeida et P. Weil, Free profinite semigroups over semidirect products, Izvestiya VUZ Matematika 39 (1995) 3-31. English version, Russian Mathem. (Iz. VUZ.) 39 (1995) 1-28.

[2] J. A. Brzozowski and I. Simon, Characterizations of locally testable languages, Discrete Math. 4, (1973), 243-271.

[3] R. S. Cohen and J. A. Brzozowski, On star-free events, Proc. Hawaii Int. Conf. on System Science, Honolulu, (1968), 1-4.

[4] J. Cohen, D. Perrin and J.-E. Pin, On the expressive power of temporal logic, J. Comput. System Sci. 46, (1993), 271-294.

[5] S. Eilenberg, Automata, languages and machines, Vol. B, Academic Press, New York, 1976.

[6] S. Emery, Varieties and pseudovarieties of ordered normal bands, Semigroup Forum, 58, (1999), 348-366. 
[7] C. Glaßer and H. Schmitz, Languages of dot-depth 3/2, Proceedings 17th Symposium on Theoretical Aspects of Computer Science, Springer Verlag, Lecture Notes in Computer Sciences 1770, (2000), 555-566.

[8] R. Knast, A semigroup characterization of dot-depth one languages, RAIRO Inform. Théor. 17, (1983) 321-330.

[9] R. Knast, Some theorems on graph congruences, RAIRO Inform. Théor. 17, (1983) 331-342.

[10] R. McNaughton, Algebraic decision procedures for local testability, Math. Syst. Theor. 8, (1974), 60-76.

[11] A. R. Meyer, A note on star-free events, J. Assoc. Comput. Mach. 16, (1969), 220-225.

[12] J.-E. Pin, Variétés de langages formels, Masson Paris, 1984; English translation: Varieties of formal languages, Plenum, New-York, 1986.

[13] J.-E. Pin, A variety theorem without complementation, Izvestiya VUZ Matematika 39 (1995) 80-90. English version, Russian Mathem. (Iz. VUZ) 39 (1995) 74-83.

[14] J.-E. Pin, Syntactic semigroups, Chapter 10 in Handbook of formal languages, G. Rozenberg and A. Salomaa eds., Springer, 1997.

[15] J.-E. Pin, A. Pinguet and P. Weil, Ordered categories and ordered semigroups, to appear in Comm. in Algebra.

[16] J.-E. Pin and P. Weil, Polynomial closure and unambiguous product, Theory Comput. Systems 30, (1997), 1-39.

[17] J.-E. Pin and P. Weil, Semidirect products of ordered semigroups, to appear in Comm. in Algebra.

[18] I. Simon, Piecewise testable events, Proc. 2nd GI Conf., Lect. Notes in Comp. Sci. 33, Springer, Berlin, 1975, 214-222.

[19] H. Straubing, Families of recognizable sets corresponding to certain varieties of finite monoids, J. Pure Appl. Algebra, 15, (1979) 305-318.

[20] H. Straubing, Finite semigroups varieties of the form $\mathbf{V} * \mathbf{D}$, J. Pure Appl. Algebra 36, (1985), 53-94. 
[21] H. Straubing, The wreath product and its application, in Formal properties of finite automata and applications, (J.-E. Pin ed.), Lect. Notes in Comp. Sci. 386, Springer Verlag, Berlin, Heidelberg, New York, (1989), $15-24$.

[22] T. Wilke, Classifying discrete temporal properties, STACS 99, Ch. Meinel and S. Tison, eds, Lect. Notes in Comput. Sci. 1563, SpringerVerlag, Berlin, (1999), 32-46. 